\title{
Chandra X-ray observation of the H II region Gum 31 in the Carina nebula complex ${ }^{\star}, \star \star$
}

\author{
T. Preibisch ${ }^{1}$, M. Mehlhorn ${ }^{1}$, L. Townsley ${ }^{2}$, P. Broos ${ }^{2}$, and T. Ratzka ${ }^{1,3}$ \\ 1 Universitäts-Sternwarte München, Ludwig-Maximilians-Universität, Scheinerstr. 1, 81679 München, Germany \\ e-mail: preibisch@usm.uni-muenchen.de \\ 2 Department of Astronomy \& Astrophysics, Pennsylvania State University, University Park PA 16802, USA \\ ${ }^{3}$ Institute for Physics / IGAM, Karl-Franzens-Universität, Universitätsplatz 5/II, 8010 Graz, Austria
}

Received 26 November 2013 / Accepted 10 March 2014

\begin{abstract}
Context. Gum 31 is a prominent, but still rather poorly studied H II region around the stellar cluster NGC 3324 at the northwestern periphery of the Carina nebula complex.

Aims. Our aim was to reveal and characterize the young stellar population in Gum 31. An X-ray survey is the only efficient way to identify young stars in this region with extremely high galactic field-star contamination that can avoid the strong biases of infrared excess selected samples of disk-bearing young stars.

Methods. We used the Chandra observatory to perform a deep (70 ks) X-ray observation of the Gum 31 region and detected 679 X-ray point sources. This extends and complements the X-ray survey of the central Carina nebula regions performed in the Chandra Carina Complex Project (CCCP). Using deep near-infrared images from our recent VISTA survey of the Carina nebula complex, our comprehensive Spitzer point-source catalog, and optical archive data, we identify counterparts for $75 \%$ of these X-ray sources.

Results. The spatial distribution of the X-ray selected young stars shows two major concentrations, the central cluster NGC 3324 and a partly embedded cluster in the southern rim of the H II region. However, these two prominent clusters contain only about $30 \%$ of the X-ray selected population, whereas the majority $(\sim 70 \%)$ of X-ray sources constitute a rather homogeneously distributed population of young stars. Our color-magnitude diagram analysis suggests ages of $\sim 1-2 \mathrm{Myr}$ for the two clusters, whereas the distributed population shows a wider age range up to $\sim 10 \mathrm{Myr}$. We also identify previously unknown companions to two of the three O-type members of NGC 3324 and detect diffuse X-ray emission in two parts of the region.

Conclusions. An extrapolation based on the observed X-ray luminosity function suggests that the observed region contains about 4000 young stars in total (down to $0.1 M_{\odot}$ ). This shows that the Gum 31 area contains a substantial fraction of the total stellar population in the CNC. The distributed population of young stars in the Gum 31 region is probably a part or extension of the widely distributed population of $\sim 1-10$ Myr old stars, that was identified in the CCCP area. This implies that the global stellar configuration of the Carina nebula complex is a very extended stellar association, in which the (optically prominent) clusters contain only a minority of the stellar population.
\end{abstract}

Key words. stars: formation - stars: pre-main sequence - X-rays: stars - open clusters and associations: individual: NGC $3324-$ open clusters and associations: individual: NGC 3372

\section{Introduction}

The Carina nebula complex (CNC) is one of the largest, most massive, and most active star-forming complexes in our Galaxy. Located at a moderate and well known distance of $2.3 \mathrm{kpc}$ (Smith 2006), the nebulosity extends over about 100 pc, corresponding to several degrees on the sky. The clouds contain a total gas and dust mass of about $10^{6} M_{\odot}$ (Preibisch et al. 2012) and harbor presumably about 100000 young stars (Feigelson et al. 2011; Povich et al. 2011; Preibisch et al. 2011c). The star formation rate of $\sim 0.01-0.02 M_{\odot} / \mathrm{yr}$ (see Povich et al. 2011; Gaczkowski et al. 2013) constitutes as much as about $1 \%$ of the total galactic star formation. The large population of massive stars ( $\geq 70$ O-type and Wolf-Rayet stars Smith 2006), including

\footnotetext{
* The Chandra data described in this paper have been obtained in the open time project with Sequence Number 200767 and ObsID 13613 (PI: T. Preibisch).

$\star \star$ Tables 1 and 3 are only available at the CDS via anonymous ftp to cdsarc.u-strasbg.fr (130.79.128.5) or via

http://cdsarc.u-strasbg.fr/viz-bin/qcat?]/A+A/564/A120
}

the object $\eta$ Car (which is the most luminous known star in our Galaxy) creates very high levels of ionizing radiation and stellar wind power, which profoundly influence the surrounding clouds. This stellar feedback has already dispersed a large fraction of the original dense molecular clouds, out of which the stars formed, but the compression of the remaining clouds by the ionization fronts and expanding wind bubbles is also currently triggering the formation of new generations of stars in the complex (Smith et al. 2010b; Gaczkowski et al. 2013).

A general review of the CNC is provided in the book chapter by Smith \& Brooks (2008). During the last five years, several new and sensitive surveys of different parts of the CNC have been performed at wavelengths from the X-ray to submm regime. One major milestone was the deep X-ray imaging survey of the Chandra Carina Complex Project (CCCP; see Townsley et al. 2011a, for an overview), which mapped an area of about 1.4 square-degrees with a mosaic of 22 individual ACIS-I pointings, using a total observing time of 1.34 Megaseconds (15.5 days). With a detection limit corresponding to X-ray luminosities of about $10^{30} \mathrm{erg} / \mathrm{s}$, these X-ray data can 
detect the coronal X-ray emission of young ( $\lesssim 10^{7} \mathrm{yrs}$ ) stars down to $\sim 0.5 M_{\odot}$. The Chandra images revealed 14368 individual X-ray sources, and a sophisticated classification scheme showed that 10714 of these are most likely young stars in the Carina nebula (Broos et al. 2011b). The analysis of the spatial distribution of the X-ray detected young stars showed that half of the young stellar population resides in one of about 30 clusters or stellar groups, while the other half constitutes a widely dispersed population (Feigelson et al. 2011) that is spread throughout the entire observed area. The combination of these CCCP X-ray data with a deep near-infrared (NIR) survey (Preibisch et al. 2011c) obtained with HAWK-I at the ESO VLT provided information about the properties of the stellar populations in the central parts of the complex (Preibisch et al. 2011b), including the individual stellar clusters $\operatorname{Tr} 16$ and $\operatorname{Tr} 15$ (Wolk et al. 2011; Wang et al. 2011).

Because of the wide spatial extent of the CNC, most recent studies (and all those mentioned above) have focused on the central $\lesssim 1.5$ square-degree area. At the northwestern periphery of the $\mathrm{CNC}$, about $80^{\prime}$ (or $\approx 50 \mathrm{pc}$ ) from $\eta \mathrm{Car}$, the $\mathrm{H}$ II region Gum 31 (first described by Gum 1955) constitutes the most prominent object in this area of the sky (see Fig. 1). Gum 31 is a roughly circular nebula with a diameter of about $12^{\prime}(\approx 8 \mathrm{pc})$, and is excited by the young stellar cluster NGC 3324 (containing three known O-type stars). Despite its interesting morphology and the publicity of Hubble Space Telescope (HST) ${ }^{1}$ and ground-based optical images ${ }^{2}$, the Gum 31 nebula (sometimes designated as the Gabriela Mistral nebula) remained quite poorly studied until very recently, probably because the closeness to the extremely eye-catching Carina nebula has always overshaded Gum 31. In the cluster NGC 3324, stellar spectral types are only known for the three optically brightest stars, HD 92206 A, B, and C, which have been classified as O6.5V (HD $92206 \mathrm{~A}$ and HD 92206 B) and O9.5V (HD 92206 C) (Mathys 1988; Walborn 1982). Carraro et al. (2001) identified 25 candidate members by means of optical photometry and suggested that the cluster age is presumably a few Myr. In addition to the optically visible cluster NGC 3324 in the H II region, a few dozen of embedded young star candidates have been found in the clouds surrounding the $\mathrm{H}$ II region, either in infrared images (Cappa et al. 2008) or traced by their protostellar jets (Smith et al. 2010a; Ohlendorf et al. 2012). However, these objects known until recently must represent only the tip of the iceberg: given the presence of three O-type stars $\left(M \geq 18 M_{\odot}\right)$, the extrapolation of the canonical stellar IMF (Kroupa 2002) would suggest a total population of $\approx 1500$ low-mass $\left(0.1 M_{\odot} \leq M \leq 2 M_{\odot}\right)$ stars. It is thus quite obvious that the vast majority of the stellar population in the Gum 31 region is still completely unknown.

While Walborn (1982) already concluded that the distance modulus values of the brightest stars in NGC 3324 are consistent with those of the clusters Tr 16 / Col 228 in the central parts of the Carina nebula, the apparent segregation of the Gum 31 nebula from the bright $\mathrm{H} \alpha$ emission of the Carina nebula in optical images left the physical relation unclear. New information on this question came from our recent Herschel far-infrared observations (Preibisch et al. 2012; Roccatagliata et al. 2013) of the $\mathrm{CNC}$. The field-of-view of this survey was wide enough (more than 5 square-degrees) to include the area around Gum 31. The Herschel maps showed that the dense dusty shell surrounding the Gum $31 \mathrm{H}$ II region is connected by numerous filamentary

\footnotetext{
1 hubblesite.org/newscenter/archive/releases/2008/34/

2 e.g., ESO Photo Release http://www.eso.org/public/news/ eso1207/
}

cloud structures to the dense molecular clouds in the inner parts of the Carina nebula, suggesting Gum 31 to be part of the CNC. The shell around Gum 31 and the surrounding clouds host several dozens of deeply embedded protostars and pre-stellar cores, which are detected as point-like sources in our Herschel data (Gaczkowski et al. 2013). In the recent study of Ohlendorf et al. (2013), these Herschel data were combined with Spitzer archive data and the Wide-field Infrared Survey Explorer (WISE) pointsource catalog and lead to the identification of some 600 young stellar object (YSO) candidates in a one square-degree area centered on Gum 31 by means of their infrared excesses. A clear concentration of partly embedded young stellar objects is located in a dense cloud at the southwestern edge of the Gum 31 bubble. This very young cluster, which we will designate as G286.38-0.26 in following text, is located at a position where the Gum 31 shell seems to interact with the expanding superbubbles driven by the numerous massive stars in the central parts of the CNC. This may represent an interesting example of triggered star formation in a cloud that formed and/or is compressed by colliding large-scale shocks.

The current, infrared-excess selected sample of protostars and disk-bearing young stars in the Gum 31 region, resulting from our analysis of the Herschel and Spitzer data (Ohlendorf et al. 2013), contains objects down to stellar masses of about $1 M_{\odot}$. However, since the stars in this region are presumably already several Myr old, this infrared-excess selected sample must be highly incomplete, because it is well known that the typical lifetime of circumstellar disks around young stars are just a few Myr (e.g., Fedele et al. 2010). The young stars which have already dispersed their disks will no longer exhibit infrared excesses and can thus not be identified by infrared excess selection. This represents a major obstacle in the identification of the young stellar population in the Gum 31 region, which is a fundamental prerequisite for an determination of its star formation history. Furthermore, because Gum 31 lies very close to the galactic plane $\left(b \approx-0.2^{\circ}\right)$ a great deal of confusion results from galactic field star contamination. All optical and infrared images of this region are thus completely dominated by unrelated field stars, and it is therefore impossible to identify and distinguish a population of several Myr old low-mass stars from unrelated field stars with optical or infrared photometry alone.

An X-ray survey, however, can solve this problem: the strongly enhanced X-ray emission of young ( $\left.\$ 10^{7} \mathrm{yrs}\right)$ stars (see, e.g., Feigelson et al. 2007; Preibisch et al. 2005) provides an extremely useful discriminant between young pre-main sequence stars and the much older field stars. The median X-ray luminosity of a few Myr old solar-mass stars is nearly 1000 times higher than for solar-mass field stars (see Preibisch \& Feigelson 2005), and makes these young stars relatively easily detectable targets for current observatories, even at the relative large distance of $2.3 \mathrm{kpc}$. This was the motivation to perform the deep Chandra X-ray observation of the Gum 31 region presented in this paper. In Sect. 2 we describe the X-ray observation and data analysis and discuss basic properties of the X-ray sources. We then describe in Sect. 3 the deep NIR data from our recent VISTA survey of the CNC and our Spitzer point-source catalog that are then used in Sect. 4 to identify and characterize the counterparts of the X-ray sources. In Sect. 5 we discuss the X-ray and infrared/optical properties of particularly interesting objects. In Sect. 6 we use near- and mid-infrared color-color and color-magnitude diagrams to infer the extinctions, infrared excesses, masses, and ages of the X-ray detected objects. Section 7 contains a discussion of the global properties of the X-ray selected population in Gum 31. In Sect. 8 we present 

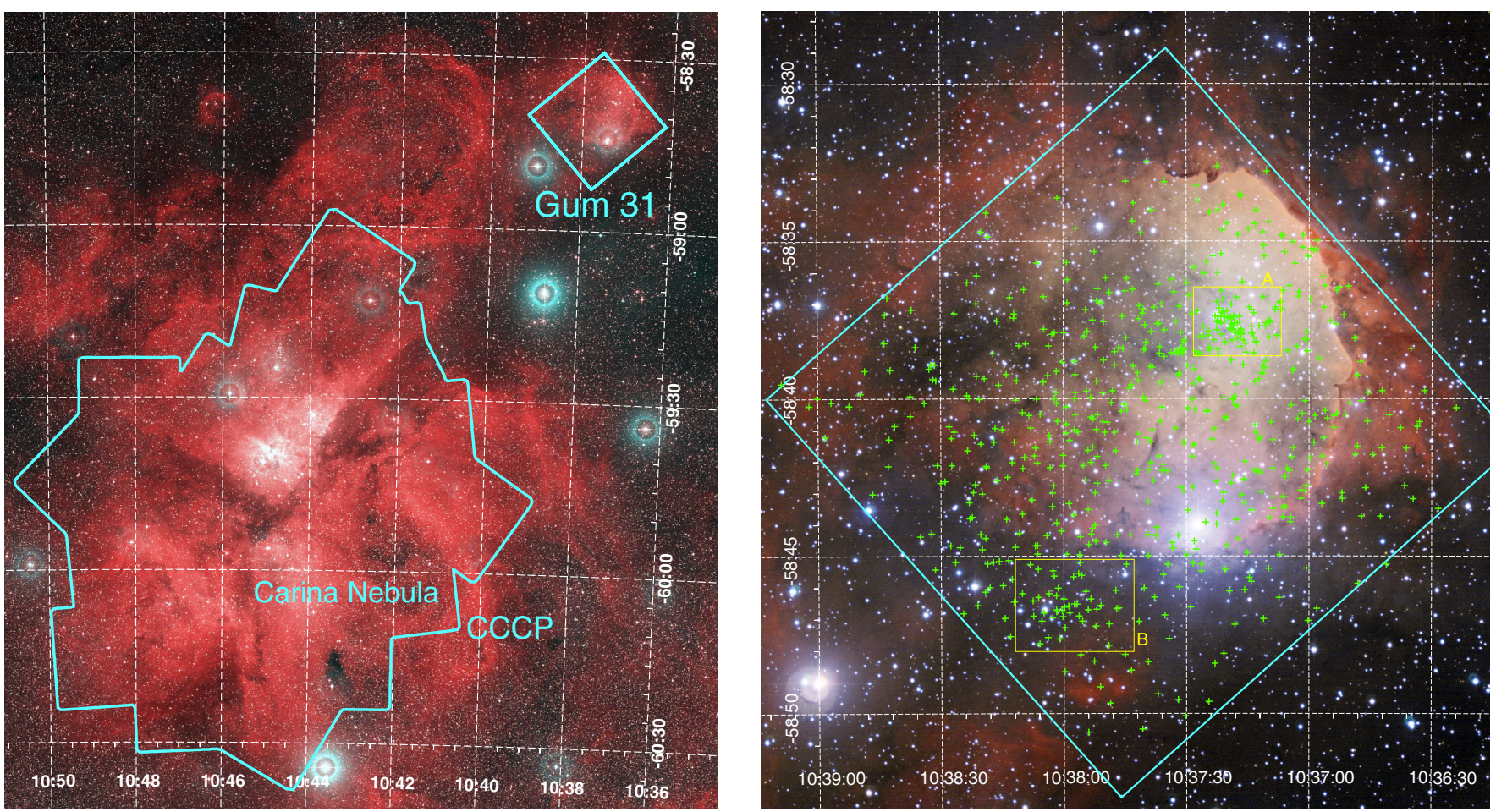

Fig. 1. Left: optical image of the Carina nebula complex (from: www. eso.org/public/images/eso0905b/; image credit: ESO/Digitized Sky Survey 2, Davide De Martin). The region observed in the context of the Chandra Carina Complex Project (CCCP) and the Chandra pointing of the Gum 31 region are marked by the cyan outlines. Right: optical image of the Gum 31 region obtained with the Wide Field Imager on the MPG/ESO $2.2 \mathrm{~m}$ telescope at La Silla Observatory (from: www. eso.org/public/images/eso1207a/; image credit: ESO) with outline of the Chandra pointing in cyan. The individual X-ray point sources are marked by green crosses. The yellow boxes mark the two regions shown in Fig. 5 (box A corresponds to the region of the cluster NGC 3324, while box B corresponds to the region of the cluster G286.38-0.26).

and briefly discuss the diffuse X-ray emission that is detected in our Chandra image. Finally, Sect. 9 contains a brief summary and the conclusions from this study.

\section{Chandra X-ray observation and data analysis}

We have used the Chandra observatory (Weisskopf et al. 2002) to perform a deep pointing of the Gum 31 region with the Imaging Array of the Chandra Advanced CCD Imaging Spectrometer (ACIS-I; see Garmire et al. 2003). The observation was performed as an open time project with sequence number 200767 and ObsID 13613 (PI: T. Preibisch) during Chandra Observing Cycle 13 in October 2012 (start date: 201210-08T20:20:42, end date: 2012-10-09T16:43:01). ACIS-I provides a field of view of $17^{\prime} \times 17^{\prime}$ on the $\mathrm{sky}^{3}$ (what corresponds to $11.3 \times 11.3 \mathrm{pc}$ at the distance of $2.3 \mathrm{kpc}$ ), and has a pixel size of $0.492^{\prime \prime}$. The point spread function of the X-ray telescope has a FWHM of $0.5^{\prime \prime}$ on-axis, but increases towards the edge of the detector. Since the Chandra aspect reconstruction errors are usually very small $\left(\lessgtr 0.1^{\prime \prime}\right)$, the positions of bright X-ray sources can usually be determined with subarcsecond precision, and sources with separations as close as $\lesssim 1^{\prime \prime}$ can be resolved.

The aimpoint of the observation was set to be $\alpha(\mathrm{J} 2000)=$ $10^{\mathrm{h}} 37^{\mathrm{m}} 36.6^{\mathrm{s}}, \delta(\mathrm{J} 2000)=-58^{\circ} 41^{\prime} 18^{\prime \prime}$. This position is close

\footnotetext{
Although two CCDs of the spectroscopic array ACIS-S were also operational during our pointing, we did not include these data in our analysis since the point-spread function is seriously degraded at the corresponding large offaxis angles. Inspection of the two ACIS-S array regions shows a high level of almost homogeneously background, but no obvious point sources.
}

to the center of the $\mathrm{H}$ II region, and allows both the stellar cluster NGC 3324 and the cluster G286.38-0.26 to be in the inner parts of the field-of-view, where the point-spread function is still very good. The pointing roll angle (i.e., the orientation of the detector with respect to the celestial north direction) was $138.35^{\circ}$. The ACIS field-of-view is just wide enough to cover the full spatial extent of the optically bright Gum $31 \mathrm{H}$ II region and some parts of the surrounding dust shell (see Fig. 1).

The observation was performed in the standard "Timed Event, Very Faint" mode with $5 \times 5$ pixel event islands. The total net exposure time of the observation was $68909 \mathrm{~s}(19.14 \mathrm{~h})$.

At the distance of $2.3 \mathrm{kpc}$, the expected ACIS point source sensitivity limit for a 5-count detection on-axis in a $70 \mathrm{ks} \mathrm{ob-}$ servation is $L_{\mathrm{X}, \min } \sim 10^{29.8} \mathrm{erg} \mathrm{s}^{-1}$, assuming an extinction of $A_{V} \leq 2.5 \mathrm{mag}\left(N_{\mathrm{H}} \leq 5 \times 10^{21} \mathrm{~cm}^{-2}\right)$ as typical for the stars in the $\mathrm{H}$ II region, and a thermal plasma with $k T=1 \mathrm{keV}$ (which is a typical value for young stars; see, e.g., Preibisch et al. 2005). Using the empirical relation between X-ray luminosity and stellar mass and the temporal evolution of X-ray luminosity from the sample of young stars in the Orion nebula cluster that was very well studied in the Chandra Orion Ultradeep Project (Preibisch et al. 2005; Preibisch \& Feigelson 2005), we can expect to detect most $(>80 \%)$ of the stars with $M \geq 1 M_{\odot}$ and about half of the $0.1 M_{\odot}<M<1 M_{\odot}$ stars in a $\leq 5$ Myr old population. These estimates are applicable to the lightly-obscured stars in NGC 3324. For the more deeply embedded sources in the denser clouds, assuming $A_{V}=7.5 \mathrm{mag}\left(N_{\mathrm{H}}=1.5 \times 10^{22} \mathrm{~cm}^{2}\right)$ and a thermal plasma with $k T=3 \mathrm{keV}$ (typical for embedded young stellar objects), the sensitivity limit increases only slightly to 
$L_{\mathrm{X} \text {,min }} \approx 10^{30.0} \mathrm{erg} \mathrm{s}^{-1}$. These limits are very similar to those of the CCCP observations of the central parts of the Carina nebula.

\subsection{X-ray point-source detection and analysis with ACIS Extract}

All details of the employed data analysis techniques are discussed in Broos et al. (2010), hereafter referred to as B10; we just provide a brief summary of this procedure here. ChandraACIS event data were calibrated and cleaned as described in Sect. 3 of B10. Those procedures seek to improve the accuracy of event properties (individual event positions, alignment of the Chandra coordinate system to an astrometric reference, energy calibration) and seek to discard events that are likely caused by various instrumental background components.

Candidate point sources were then identified in the pointing using two methods, the standard Chandra detection tool, wavdetect (Freeman et al. 2002) and a Lucy-Richardson image reconstruction (Lucy 1974), that were performed on images in different energy bands and pixels sizes. These candidate sources were then extracted using the ACIS Extract (hereafter AE) software package $^{4}$ (Broos et al. 2012).

For each source, AE calculated the probabilities that the counts extracted in each of three energy bands arose solely from the local background. When all three probabilities were greater than 0.01 or when less than three X-ray counts were extracted, we judged the candidate source to be not significant, and removed it from the list. The positions of surviving source candidates were updated with AE estimates, and the reduced list of candidates was re-extracted. This cycle of extraction, pruning, and position estimation was repeated until no candidates were found to be insignificant.

Our final X-ray catalog contains 679 individual point sources. The number of extracted counts ranges from 3 for the faintest sources, up to 920 for the strongest source; the median value is 11 counts. AE screened all sources for an instrumental non-linearity known as photon pile-up ${ }^{5}$; no sources were found to be at risk of pile-up.

The final list of X-ray sources with their properties is reported in Table 1 (available in the electronic edition). Sources are sorted by increasing right ascension and identified by their sequence number (Col. 1) or their IAU designation (Col. 2). Following the rules for the designation of sources found with the Chandra X-ray Observatory, we have registered the acronym CXOPMTB as the prefix for the IAU designation (Col. 2).

\subsection{X-ray source variability}

The AE procedure investigates the time variability of each X-ray source by comparing the arrival times of the individual source photons in each extraction region to a model assuming temporal uniform count rates. The statistical significance for variability is then computed with a 1-sided Kolmogorov-Smirnov statistic (Col. 15 of Table 1). In our sample, 42 sources show significant X-ray variability (probability of being constant $P_{\text {const }}<0.005$ ) and additional 48 sources are classified as possibly variable $\left(0.005<P_{\text {const }}<0.05\right)$.

The lightcurves of the variable X-ray sources show a variety of temporal behavior; six of the most interesting lightcurves are

\footnotetext{
4 The ACIS Extract software package and User's Guide are available at http://www . astro.psu.edu/xray/acis/acis_analysis.html

5 http://cxc.harvard.edu/ciao/why/pileup_intro.html
}

shown in Fig. 2. Three of these sources show flare-like variability, i.e., a fast increase in the count rate followed by a slow exponential decay, as typical for solar-like magnetic reconnection flares (see, e.g., Wolk et al. 2005). The other variable sources show more slowly increasing or decreasing count rates, as also often found for young stellar objects (see, e.g., Stassun et al. 2006).

\subsection{X-ray spectral fits of bright sources}

For the 22 sources in our sample with more than 100 net counts we performed a spectral fitting analysis using the SHERPA v4.5 software in CIAO (see Freeman et al. 2001). SHERPA includes the plasma emission and absorption models of XSpec version 11.3. The background-subtracted spectra were first grouped into bins containing at least 10 counts, and the fits were then performed using the $\chi^{2}$ statistic with the Gehrels (1986) variance function $^{6}$, which is the SHERPA default.

We used models with one or more thermal plasma VAPEC components, and the TBABS model to describe the effect of extinction by interstellar and/or circumstellar material (as parameterized by the hydrogen column density $N_{\mathrm{H}}$ ). The elemental abundances for the plasma model were fixed at the values ${ }^{7}$ that were found by Güdel et al. (2007) to be typical for young stellar objects.

Most spectra could be well reproduced by one-temperature models. The only case where models with more than one temperature components produced significantly better fits was the O star HD 92206 A, which has 920 source counts (this X-ray spectrum is discussed in more detail in Sect. 5). A few selected examples of the spectral fits are shown in Fig. 3, while the resulting best fit spectral parameters for all sources are reported in Table 2. We also list there the intrinsic (i.e., extinction corrected) X-ray luminosities $L_{\mathrm{X}, \mathrm{tc}}$ for the total $(0.5-8 \mathrm{keV})$ band, as derived from the spectral fit parameters.

The range of X-ray luminosities spans from $\log \left(L_{\mathrm{X}, \mathrm{tc}}[\mathrm{erg} / \mathrm{s}]\right)=30.73$ to 31.88 . The hydrogen column densities derived in the fits are mostly around $N_{\mathrm{H}} \sim 0.3 \times 10^{22} \mathrm{~cm}^{-2}$, corresponding to visual absorptions of $A_{V} \sim 1.5$ mag. Only a few sources show column densities above $10^{22} \mathrm{~cm}^{-2}$ ( $\left.A_{V} \gtrsim 5 \mathrm{mag}\right)$. The highest extinction of $N_{\mathrm{H}}=3.6 \times 10^{22} \mathrm{~cm}^{-2}$ ( $A_{V} \sim 18 \mathrm{mag}$ ) is found for the source J103758.57-584647.9, a faint infrared source in the dense cloud associated to the cluster G286.38-0.26. These values are in good agreement with the cloud column densities derived from our Herschel far-infrared data (see Preibisch et al. 2012; and Fig. 4 in Ohlendorf et al. 2013).

The derived plasma temperatures range from $\approx 0.57 \mathrm{keV}$ ( $\approx 7 \mathrm{MK}$ ) for J103718.63-583741.9, up to $\sim 7.29 \mathrm{keV}(\approx 85 \mathrm{MK})$ for J103701.61-583512.0. The later source is a faint, optically visible star near the northwestern edge of the $\mathrm{H}$ II region, which showed a strong flare during our Chandra observation (see lightcurve in Fig. 2).

The derived plasma temperatures and X-ray luminosities are in the typical ranges found for YSOs in other star forming

\footnotetext{
6 The standard deviation is calculated with the formula $\sigma=1+$ $\sqrt{N+0.75}$, which is thought to be more realistic than assuming a Poisson distribution, if the number of counts per bin is low. We note that for spectra with 10 counts per bin, the expected value for the reduced chi-square statistic with the Gehrels variance is $E\left[\chi_{n}^{2}\right]=0.74$.

7 The adopted abundances, relative to the solar photospheric abundances given by Anders \& Grevesse (1989), are: $\mathrm{C}=0.45, \mathrm{~N}=0.788$, $\mathrm{O}=0.426, \mathrm{Ne}=0.832, \mathrm{Mg}=0.263, \mathrm{Al}=0.5, \mathrm{Si}=0.309, \mathrm{~S}=0.417$, $\mathrm{Ar}=0.55, \mathrm{Ca}=0.195, \mathrm{Fe}=0.195, \mathrm{Ni}=0.195$.
} 

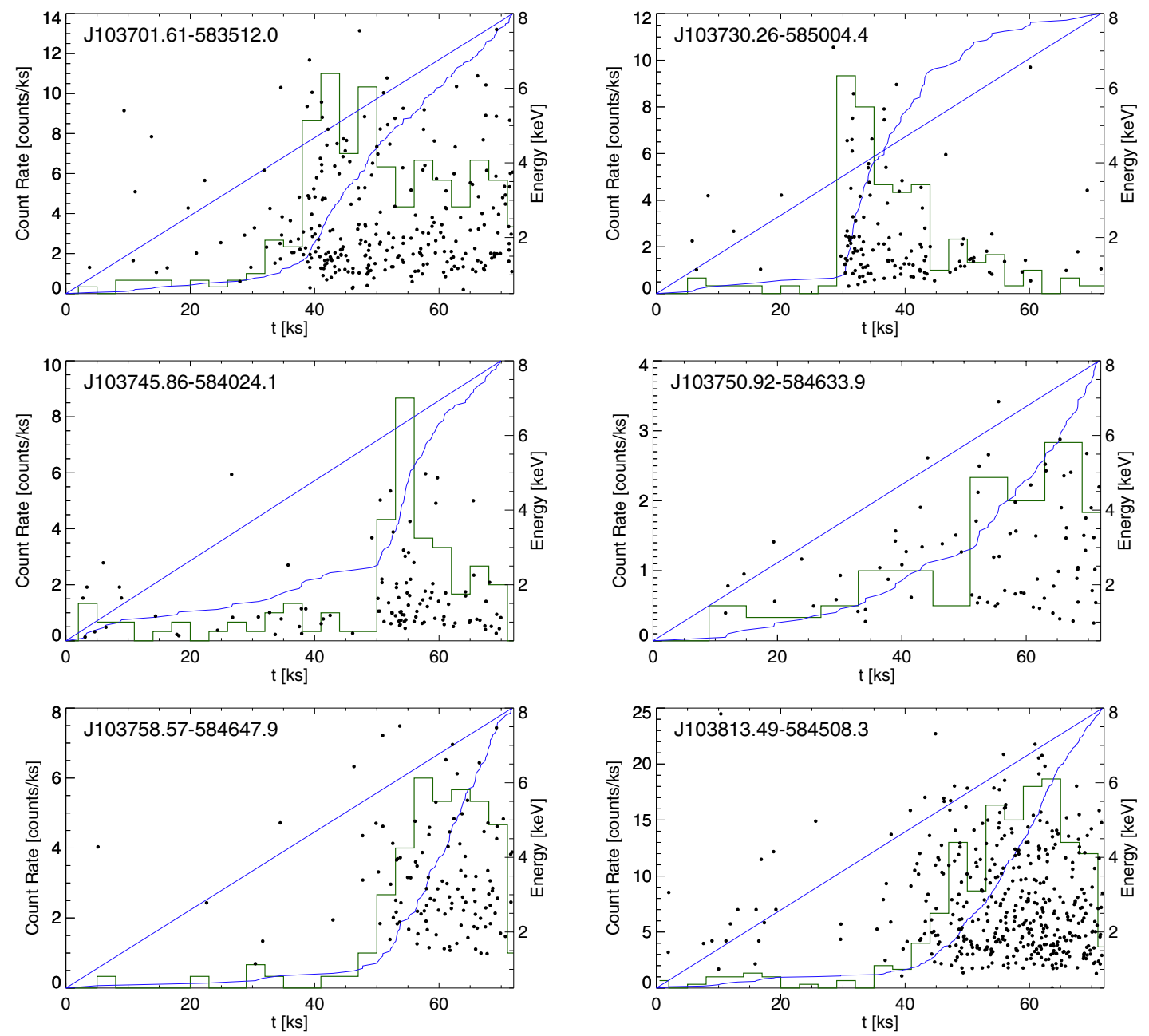

Fig. 2. Lightcurves for six significantly variable sources. The solid dots show the arrival time (measured from the start of the observation) and the energy of each of the detected source photons. The histograms show the corresponding binned lightcurves. The solid lines show the cumulative distribution function of the photon arrival times compared to the expectation for a perfectly constant source (the diagonal line).

regions (see, e.g., Preibisch et al. 2005). A detailed discussion of individual interesting sources will be given in Sect. 5 .

\subsection{X-ray luminosity estimates for fainter sources}

Most of the X-ray sources have less than 100 source counts, which is a practical limit for detailed X-ray spectral analysis. For these fainter sources, we used the XPHOT software $^{8}$, developed by Getman et al. (2010), to derive an estimate of the intrinsic (i.e., extinction corrected) X-ray luminosity of the X-ray sources. XPHOT is based on a non-parametric method for the calculation of fluxes and absorbing X-ray column densities of weak X-ray sources. X-ray extinction and intrinsic flux are estimated from the comparison of the apparent median energy of the source photons and apparent source flux with those of high signal-to-noise spectra that were simulated using spectral models characteristic of much brighter sources of similar class previously studied in detail. This method requires $\geq 5$ net counts per source and can thus be applied to 286 of our 679 sources. For the remaining sources, the "energy flux" determined by ACIS Extract can be used to compute a rough estimate of the X-ray luminosity (see discussion in Broos et al. 2011a, for more details). For those 22 source for which a spectral fit was performed,

\footnotetext{
8 www.astro.psu.edu/users/gkosta/XPHOT/
}

a comparison shows that the XPHOT and the spectral fit X-ray luminosity estimates agree within a factor of two in most cases.

\subsection{Spatial distribution of the $X$-ray sources}

We performed a nearest neighbor analysis (see Casertano \& Hut 1985) to obtain a quantitative characterization of the spatial distribution of X-ray sources. The nearest neighbor technique allows statistically significant overdensities to be identified in an objective way and is widely used in studies of the clustering properties of star forming regions (see, e.g., Gutermuth et al. 2009). In the present study, we used the distance to the fifth nearest neighbor to calculate the surface density estimator $\mu_{5}$ at the position of each source. For the interpretation of the resulting densities, we have to take the spatial variations of the detection sensitivity over the ACIS field-of-view into account: sensitivity is highest in the center, and decreases (due to factors such as mirror vignetting and the increasing width of the point-spread function) towards the edges of the field-of-view. We therefore plot in Fig. 4 the surface density estimator $\mu_{5}$ as a function of the offaxis-angle. The general trend of decreasing source density with increasing offaxis-angle above about $5^{\prime}$ can be clearly seen. Clusters of X-ray sources can be identified as spatially confined groups of sources for which the local surface density 

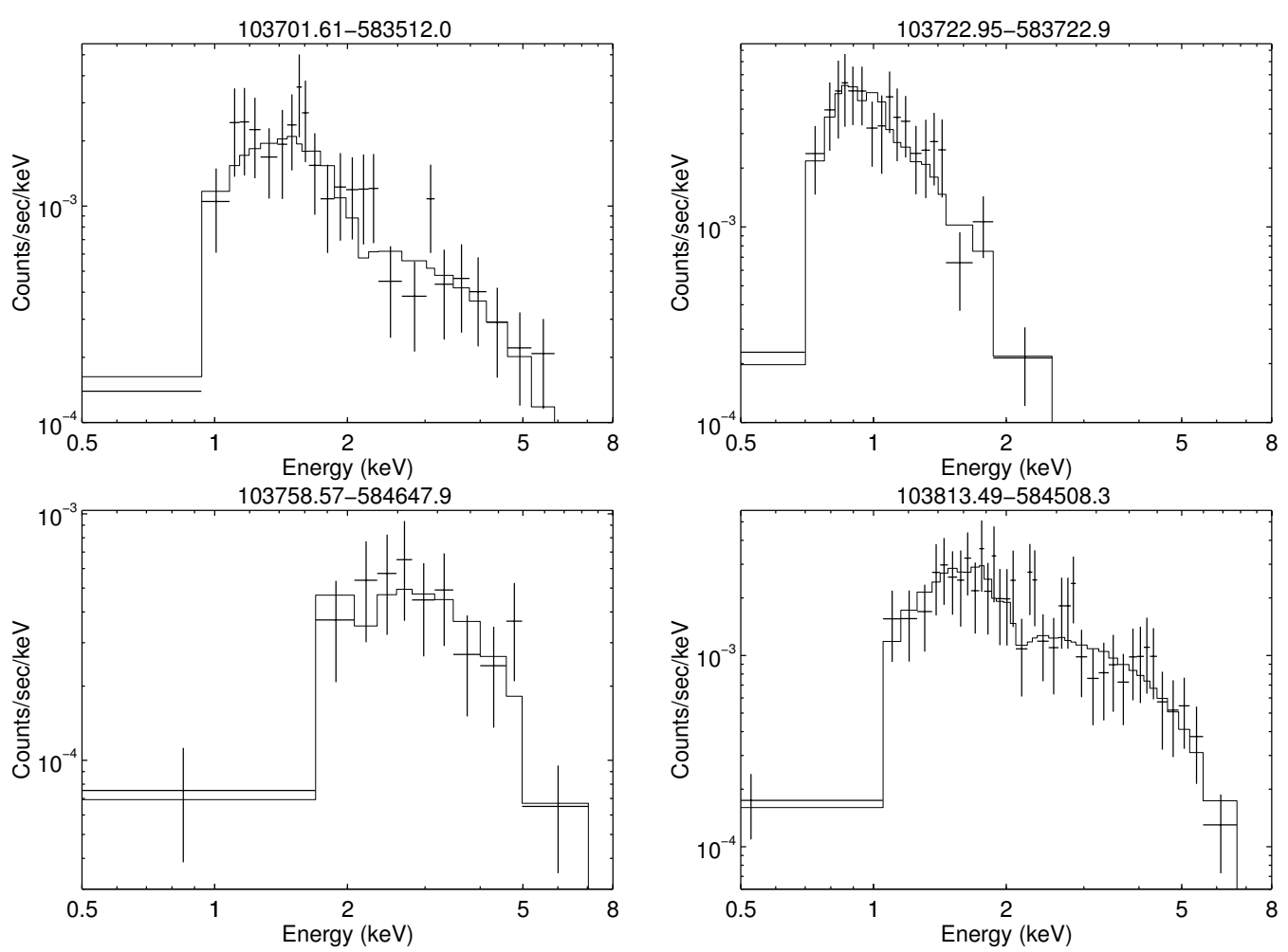

Fig. 3. Chandra X-ray spectra and best-fit models of four bright X-ray sources in our Gum 31 observation. The crosses show the measured spectra, the solid lines show the best-fit models.

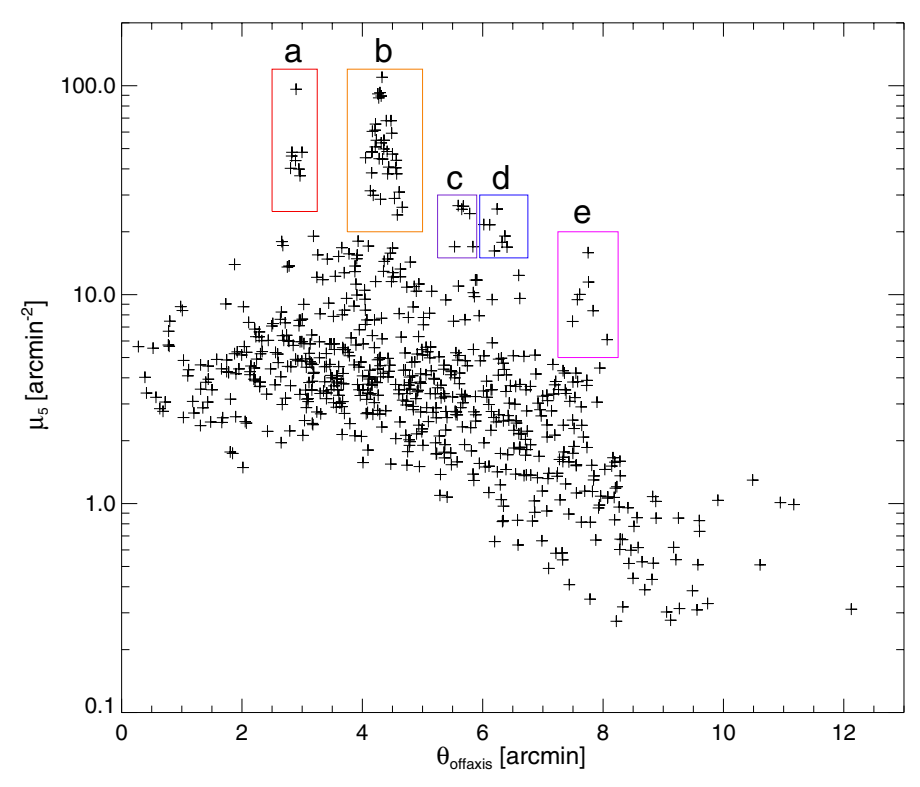

Fig. 4. Nearest neighbor surface density $\mu_{5}$ at the location of each X-ray source plotted against the offaxis-angle. The general decrease in density with increasing offaxis-angle is related to instrumental effects. The boxes enclose the members of the identified clusterings.

clearly exceeds the values found at other locations in the image at similar offaxis-angles.

We identify five different clusterings, a to e, in Fig. 4. Clustering a ( 8 members) is a rather small, but quite dense group of stars, which is located about $1.5^{\prime}$ southeast of the cluster NGC 3324 at the coordinates $\alpha(\mathrm{J} 2000)=10^{\mathrm{h}} 37^{\mathrm{m}} 31^{\mathrm{s}}$, $\delta(\mathrm{J} 2000)=-58^{\circ} 38^{\prime} 30^{\prime \prime}$. Clustering b (36 members) corresponds to the stellar cluster NGC 3324. Clustering c
(6 members) corresponds to a small, but dense group of stars, about $1^{\prime}$ northwest of the cluster NGC 3324, at the coordinates $\alpha(\mathrm{J} 2000)=10^{\mathrm{h}} 37^{\mathrm{m}} 07^{\mathrm{s}}, \delta(\mathrm{J} 2000)=-58^{\circ} 37^{\prime} 00^{\prime \prime}$. The group $\mathrm{d}$ ( 7 members) corresponds to the cluster G286.38-0.26 in the southern parts of the ACIS image. The group e ( 7 members) corresponds to the a group of stars at the northwestern inner rim of the HII region, at the coordinates $\alpha(\mathrm{J} 2000)=10^{\mathrm{h}} 36^{\mathrm{m}} 59^{\mathrm{s}}$, $\delta(\mathrm{J} 2000)=-58^{\circ} 35^{\prime} 23^{\prime \prime}$.

\subsection{Expected contamination of the $X$-ray source sample}

As in any X-ray observation, there will be some degree of contamination by galactic field stars in the fore- and background as well as extragalactic sources. To quantify the expected level of this contamination, we use here the results from the CCCP that observed the central parts of the CNC with very similar exposure times $(\approx 60-80 \mathrm{ks})$ as our Gum 31 pointing. Since our Gum 31 pointing is very close to the sky region covered by the CCCP, the contamination levels should be quite similar.

The detailed simulations of contaminating X-ray-emitting populations by Getman et al. (2011) predicted $\sim 5000$ contaminating sources ${ }^{9}$ in the CCCP field. This would imply that about $35 \%$ of all X-ray sources in the CCCP field are contaminants. The classification study of Broos et al. (2011b), which considered the X-ray, optical, and infrared properties of the sources (that differ for the different contaminant classes), found that $75 \%$ of CCCP sources can be classified as young stars in the Carina nebula, while $11 \%$ were classified as contaminants, and $14 \%$ remained unclassified (e.g., due to incomplete and/or ambiguous data). This leads to a slightly lower estimate of the contamination rate, between $\geq 11 \%$ and $\sim 25 \%$.

\footnotetext{
9 The specific predictions were $\sim 1800$ foreground stars, $\sim 900$ background stars, and $\sim 2300$ AGN.
} 
Table 2. Spectral fitting results for the $22 \mathrm{X}$-ray sources with more than 100 counts.

\begin{tabular}{lccccc}
\hline \hline Source & $\chi_{n}^{2}$ & $\begin{array}{c}N_{\mathrm{H}} \\
{\left[10^{22} \mathrm{~cm}^{-2}\right]}\end{array}$ & $\begin{array}{c}k T \\
{[\mathrm{keV}]}\end{array}$ & $\begin{array}{c}\text { norm } \\
{\left[10^{-19} \mathrm{~cm}^{-5}\right]}\end{array}$ & $\begin{array}{c}\log \left(L_{X, \mathrm{tc}}\right) \\
{[\mathrm{erg} / \mathrm{s}]}\end{array}$ \\
\hline $\mathrm{J} 103652.59-583627.8$ & 0.25 & $0.33 \pm 0.33$ & $1.15 \pm 0.41$ & $4.08 \pm 0.29$ & $31.06 \pm 0.16$ \\
$\mathrm{~J} 103653.22-583633.2$ & 0.89 & $1.80 \pm 0.51$ & $1.99 \pm 0.47$ & $8.99 \pm 3.06$ & $31.24 \pm 0.23$ \\
$\mathrm{~J} 103653.40-583737.5$ & 0.83 & $0.56 \pm 0.30$ & $0.96 \pm 0.35$ & $6.73 \pm 3.92$ & $31.16 \pm 0.33$ \\
$\mathrm{~J} 103700.41-583829.2$ & 0.14 & $0.13 \pm 0.14$ & $2.73 \pm 0.71$ & $1.77 \pm 0.42$ & $30.96 \pm 0.15$ \\
$\mathrm{~J} 103701.61-583512.0$ & 0.54 & $0.27 \pm 0.12$ & $7.29 \pm 3.53$ & $4.68 \pm 0.65$ & $31.56 \pm 0.12$ \\
$\mathrm{~J} 103706.34-583633.7$ & 0.67 & $0.26 \pm 0.20$ & $3.03 \pm 1.25$ & $9.83 \pm 3.27$ & $31.73 \pm 0.14$ \\
$\mathrm{~J} 103716.31-583617.4$ & 0.74 & $0.25 \pm 0.19$ & $4.19 \pm 3.78$ & $2.93 \pm 0.89$ & $31.26 \pm 0.18$ \\
$\mathrm{~J} 103718.63-583741.9$ & 0.55 & $0.26 \pm 0.17$ & $0.57 \pm 0.14$ & $3.68 \pm 1.87$ & $30.98 \pm 0.29$ \\
$\mathrm{~J} 103719.02-583749.1$ & 0.57 & $0.43 \pm 0.27$ & $1.37 \pm 0.52$ & $3.02 \pm 1.31$ & $30.90 \pm 0.22$ \\
$\mathrm{~J} 103722.56-583655.6$ & 0.67 & $0.43 \pm 0.15$ & $2.45 \pm 0.54$ & $3.27 \pm 0.67$ & $31.13 \pm 0.13$ \\
$\mathrm{~J} 103722.95-583722.9$ & 0.53 & $0.20 \pm 0.09$ & $0.68 \pm 0.08$ & $4.70 \pm 1.52$ & $31.21 \pm 0.13$ \\
$\mathrm{~J} 103730.26-585004.4$ & 0.60 & $0.00 \pm 0.14$ & $2.51 \pm 1.67$ & $2.27 \pm 0.55$ & $31.11 \pm 0.09$ \\
$\mathrm{~J} 103736.29-583929.4$ & 0.43 & $0.84 \pm 0.42$ & $5.32 \pm 11.1$ & $3.23 \pm 1.56$ & $31.28 \pm 0.20$ \\
$\mathrm{~J} 103745.86-584024.1$ & 0.25 & $0.00 \pm 0.12$ & $1.95 \pm 0.76$ & $1.41 \pm 0.34$ & $30.89 \pm 0.11$ \\
$\mathrm{~J} 103752.94-584407.2$ & 0.10 & $0.39 \pm 0.15$ & $2.40 \pm 0.90$ & $3.19 \pm 0.79$ & $31.13 \pm 0.19$ \\
$\mathrm{~J} 103754.06-584642.3$ & 0.42 & $1.48 \pm 0.47$ & $3.86 \pm 3.31$ & $5.66 \pm 2.42$ & $31.35 \pm 0.26$ \\
$\mathrm{~J} 103758.57-584647.9$ & 0.55 & $3.62 \pm 0.33$ & $2.61 \pm 3.12$ & $10.0 \pm 9.84$ & $31.24 \pm 0.35$ \\
$\mathrm{~J} 103807.71-584313.0$ & 0.83 & $0.00 \pm 0.02$ & $1.00 \pm 0.08$ & $2.12 \pm 0.23$ & $31.08 \pm 0.05$ \\
$\mathrm{~J} 103813.49-584508.3$ & 0.43 & $0.95 \pm 0.15$ & $6.59 \pm 1.56$ & $10.6 \pm 1.28$ & $31.80 \pm 0.09$ \\
$\mathrm{~J} 103814.39-584658.3$ & 0.44 & $0.35 \pm 0.15$ & $0.79 \pm 0.14$ & $3.65 \pm 1.24$ & $30.73 \pm 0.18$ \\
$\mathrm{~J} 103816.23-584235.8$ & 0.26 & $0.09 \pm 0.11$ & $0.76 \pm 0.12$ & $3.00 \pm 0.99$ & $31.08 \pm 0.20$ \\
\hline J103722.27-583723.0 & 0.81 & $0.31 \pm 0.06$ & $0.73 \pm 0.06$ & $22.7 \pm 3.79$ & $31.78 \pm 0.12$ \\
(1 temperature fit) & & & & & \\
J103722.27-583723.0 & 0.56 & $0.32 \pm 0.14$ & $0.38 \pm 0.05$ & $28.7 \pm 15.0$ & \\
(2 temperature fit) & & + & $2.32 \pm 1.14$ & $6.55 \pm 2.11$ & $31.85 \pm 0.21$ \\
J103722.27-583723.0 & 0.49 & $0.46 \pm 0.46$ & $0.20 \pm 0.08$ & $60.2 \pm 56.9$ & \\
(3 temperature fit) & & + & $0.54 \pm 0.54$ & $24.9 \pm 5.61$ & \\
\hline & & + & $5.17 \pm 5.17$ & $3.21 \pm 1.70$ & $31.88 \pm 0.24$ \\
\hline
\end{tabular}

Notes. The table lists for each source the quality of the fit expressed by the $\chi_{n}^{2}$, the absorbing hydrogen column density $N_{\mathrm{H}}$, the plasma temperature $k T$, the normalization (which is the emission measure of the plasma scaled by the distance $D$, i.e., norm $=\int n_{\mathrm{e}} n_{\mathrm{H}} \mathrm{d} V /\left[4 \pi D^{2}\right]$ ), and finally the intrinsic (i.e., extinction corrected) X-ray luminosity in the total $(0.5-8 \mathrm{keV})$ band, $L_{\mathrm{X}, \mathrm{tc}}$. For the brightest source J103722.27-583723.0, which corresponds to the O6.5 star HD 92206 A, we list the parameters for a single plasma temperature model fit and for two- and three-temperature model fits; these fits are discussed in 5.2.1.

Considering these two different estimates, we therefore assume a contamination rate of about $25-30 \%$ for the X-ray sources in our Gum 31 pointing, and can thus expect $\sim 180$ contaminants and $\sim 500$ young stars in our sample of 679 X-ray sources.

\section{Optical and infrared data}

Here we describe the data sets which we used for the identification and characterization of the X-ray sources.

\subsection{Optical images}

In order to search for possible optical counterparts of the X-ray sources, we first inspected optical images from the Digitized Sky Survey as well as $V$ - and $R$-band images obtained with the Wide Field Imager on the MPG/ESO $2.2 \mathrm{~m}$ telescope (which are deeper and provide better angular resolution than the DSS images). This search revealed possible optical counterparts to 371 $(54.6 \%)$ of the X-ray sources.

We also inspected the available HST archive images in the Gum 31 region. A mosaic consisting of a $\approx 3.25^{\prime} \times 6.5^{\prime}$ wide box covering the western part of the ionization front and a (partly overlapping) $\approx 3.25^{\prime} \times 3.25^{\prime}$ box covering the northern half of the cluster NGC 3324 had been obtained with ACS/WFC in the context of the 10th anniversary of the Hubble Heritage Project ${ }^{10}$. These HST images cover a total area of about 30 squarearcminutes, i.e., only about $10 \%$ of the field-of-view of our Chandra observation. $123 \mathrm{X}$-ray sources are located in the area covered by the HST images, and 84 of these (i.e., 68.3\%) have an optical counterpart.

In total, 382 of the 679 X-ray sources have an optical counterpart in the DSS, WFI and/or HST images. It is immediately clear that a reasonably complete optical characterization of low-mass stars in the Gum 31 would require much deeper optical data than available: assuming an age of about $3 \mathrm{Myr}$ and a typical extinction of $A_{V}=2 \mathrm{mag}$, stars with masses of $[1.0,0.5,0.1] M_{\odot}$ are predicted to have visual magnitudes of $V=[19.3,21.0,26.3]$ according to the pre-main-sequence stellar models of Siess et al. (2000). This shows that the majority of the low-mass $\left(\leq 1.0 M_{\odot}\right)$ stellar population will thus remain undetectable in the available optical images.

The NIR regime is much better suited to detect and characterize the young low-mass stars: for the above mentioned stellar masses, ages, and extinctions, the expected magnitudes are $J=[15.7,16.7,18.0]$. These stars will be rather easily

\footnotetext{
10 See http://hubblesite.org/newscenter/archive/ releases/2008/34/. A description of these data and the available high-level products sets can be found at http://archive. stsci.edu/prepds/carina/
} 
detectable in even relatively moderately deep NIR images (although not in the 2MASS data).

\subsection{VISTA NIR survey data}

We have recently used the $4 \mathrm{~m}$ Visible and Infrared Survey Telescope for Astronomy (VISTA; Emerson et al. 2006) at the European Southern Observatory under program number 088.C0117 (A) to obtain a deep and very wide $\left(\approx 2.3^{\circ} \times 2.9^{\circ}\right)$ NIR survey that covers the full spatial extent of the $\mathrm{CNC}$, including the entire area of Gum 31. The results of this survey are very well suited for an identification and characterization of the counterparts of the X-ray sources in Gum 31. While a detailed description of our infrared survey and the resulting source catalog will be given in Preibisch et al. (in prep.), the main aspects can be summarized as follows: we obtained deep VISTA images in the NIR $J$-, $H$-, and $K_{\mathrm{s}}$-bands during March 2012. VISTA's infrared camera VIRCAM (Dalton et al. 2006) consists of an array of sixteen individual $2048 \times 2048$ pixel infrared detectors with a nominal mean pixel size of $0.339^{\prime \prime}$ on the sky. In order to account for the gaps between the individual detectors, six individual exposures with corresponding offset in $x$ - and $y$-direction are combined to yield a so-called 'tile', that covers an area of $1.2^{\circ} \times 1.5^{\circ}$ without gaps. The entire area of our Gum 31 Chandra pointing lies within the northwestern tile of our $2 \times 2$ tile mosaic VISTA survey. These data were processed by the VISTA data flow system (see Irwin et al. 2004) at the Cambridge Astronomy Survey Unit, which provided catalogs of all detected point-like sources. The photometric calibration was performed by using stars in the 2MASS Point Source Catalog, which are present in large numbers (several thousands) in each VISTA tile, as will be described in detail in Preibisch et al. (in prep.). The final photometric uncertainties, characterized by the standard deviations between the calibrated VISTA magnitudes and 2MASS magnitudes for stars in the magnitude intervals of $\left(J, H, K_{\mathrm{S}}\right) \sim[12 \ldots 13]$ are in the range $\sigma=[0.04 \ldots 0.05]$ mag for all three bands. For the bright stars, that are in or close to the non-linear or saturated regime in at least one of the three VISTA bands (i.e., $J \leq 12.0$, $H \leq 11.75$, or $K_{\mathrm{s}} \leq 11.5$ ), photometry from the 2MASS Point Source Catalog was used.

Our final VISTA catalog contains more than four million individual sources, 3951580 of which are detected in at least two of the three NIR bands. The formal $5 \sigma$ detection limit for pointlike sources in the Gum 31 area (calculated from the measured skynoise in the corresponding tiles) is $J \approx 20.4, H \approx 19.4$, and $K_{\mathrm{S}} \approx 18.8$. Typical values for the completeness limit across the field are $J_{\text {compl }} \approx 18.5, H_{\text {compl }} \approx 18$, and $K_{\text {s, compl }} \approx 17.5$; nearly all objects brighter than these limits are $S / N \geq 10$ detections. Comparing these numbers to the above mentioned pre-main sequence models, we find that our VISTA catalog should be complete for moderately-obscured $\left(A_{V} \lesssim 5 \mathrm{mag}\right.$ ) young ( $\$ 3 \mathrm{Myr}$ ) stars down to masses of $\approx 0.1 M_{\odot}$. The number of cataloged NIR sources in the area of our Gum 31 Chandra observation is about 46000 .

As any large astronomical source catalog, the VISTA cata$\log$ is not $100 \%$ perfect. Especially in regions with strong diffuse nebulosity and near the extended point-spread-functions of very bright stars, the detection efficiency is limited and some point-like sources that are clearly visible in the images were not detected by the VISTA data processing pipeline and are thus missing from the catalog. Although this problem concerns only a very small fraction (about 1-2\%) of the total survey area, a few infrared counterparts of X-ray sources in the Gum 31 area are missing from the catalog. However, as described below, these cases could be easily identified and solved by visual inspection of the original VISTA images.

\subsection{Spitzer mid-infrared data}

For the identification of mid-infrared counterparts of the X-ray sources, we used our Spitzer point source catalog that we created from all available IRAC archive data of the CNC as described in Ohlendorf et al. (2014). The estimated completeness limits of our IRAC catalog are $\approx 1.5 \mathrm{mJy}, \approx 0.7 \mathrm{mJy}, \approx 1.2 \mathrm{mJy}$, and $\approx 1.6 \mathrm{mJy}$ for the IRAC $1,2,3$, and 4 bands. With these limits, the photospheres of young ( $\$ 3 \mathrm{Myr}$ ) stars with masses down to $\approx 0.5 M_{\odot}$ can be detected (at least) in the IRAC 1 and 2 bands, for moderate extinction $\left(A_{V} \lesssim 5 \mathrm{mag}\right)$. There are about 4300 Spitzer catalog sources in the area of the Chandra observation of Gum 31.

\subsection{Herschel far-infrared data}

Maps of the far-infrared emission in the CNC were obtained in December 2010 in our Herschel open time project. We used the PACS/SPIRE parallel mode to produce maps in the 70, 160, 250, 350 , and $500 \mu \mathrm{m}$ band for a more than 5 square-degree wide area that covers the full spatial extent of the CNC and includes the Gum 31 area. A full description of these observations and the data processing can be found in Preibisch et al. (2012). The angular resolution of the Herschel maps ranges from $5^{\prime \prime}$ in the $70 \mu \mathrm{m}$ map to $25^{\prime \prime}$ in the $500 \mu \mathrm{m}$ map.

Our Herschel images revealed 642 reliably detected pointlike sources, which have been analyzed in Gaczkowski et al. (2013). As described there in detail, the sensitivity limit of these data maps allows the detection of pre-stellar cores with cloud masses of $\gtrsim 1 M_{\odot}$ and Class 0 protostars with stellar masses of $\gtrsim 1 M_{\odot}$. Young stellar objects in later evolutionary phases are only detected as long as they still have rather massive circumstellar envelopes and/or disks; solar-mass objects will be detected only during their class I phase, but not in later phases. Intermediate mass objects with disks may also be detected during their class II phases.

There are 35 Herschel point-like sources in the field of our Chandra observation of Gum 31.

\section{Infrared counterparts of the X-ray sources}

\subsection{Source matching procedure}

In order to identify infrared counterparts of the X-ray sources in our VISTA and Spitzer catalogs, we performed a two-step process. The first step is an automatic matching that identifies counterparts solely based on the X-ray and infrared source coordinates. The second step included a detailed individual inspection of all X-ray source positions in the infrared images and also utilized the available a priori information to resolve problematic cases such as multiple possible matches.

For the first step, the automatic source matching, we employed the method described by Broos et al. (2011a) as implemented in the IDL tool ${ }^{11}$ match_xy.pro. The maximum acceptable separation between an X-ray source and a counterpart is based on the individual source position errors assuming Gaussian distributions, scaled so that $\sim 99 \%$ of true associations

\footnotetext{
11 See www2.astro.psu.edu/xray/docs/TARA/TARA_users_ guide/node11.html
} 
should be identified as matches. The X-ray source position errors determined by AE range from 0.04 " to $1.45^{\prime \prime}$, with a mean value of $0.46^{\prime \prime}$. For the VISTA sources, we assumed position uncertainties of 0.1", while for the Spitzer sources, we used the magnitude-dependent position uncertainties up to $0.5^{\prime \prime}$ as derived by the MOPEX software (see Ohlendorf et al. 2013, for details).

In the first stage, the algorithm tests the hypothesis that a possible pair of sources from the two catalogs is spatially coincident. The most significant match of each master source is referred to as its "Primary Match"; any other significant matches are "Secondary Matches". The second stage of the algorithm resolves possible many-to-one and one-to-many relationships between the X-ray catalog and the infrared catalogs. Clear one-toone relationships are classified as "successful primary matches", while in cases where, e.g., two X-ray sources are significantly close to a single infrared source, the less significant primary match is labeled as "failed". This finally provides a reasonable one-to-one set of matches.

\subsection{Chandra - VISTA matching results}

The matching procedure yielded 481 successful primary VISTA matches to the 679 Chandra sources. There was no case of failed primary matches, but for $16 \mathrm{X}$-ray sources one or more successful secondary matches were identified. These $16 \mathrm{X}$-ray sources have more than one possible infrared counterpart within the matching box, and it is not guaranteed that the closest match is always the correct physical counterpart. Since the surface density of infrared sources in our deep VISTA images is rather high $^{12}$, it is possible that physically unrelated (e.g., background) infrared sources may appear in the matching region just by chance and produce a "false match", which even could degrade the true infrared counterpart to a secondary match.

We can use a priory information about the infrared properties of the X-ray detected young stars to identify possible cases where this problem occurs. Since we know from the X-ray detection limit that most of the X-ray detected objects should be young stars with masses of $\gtrsim 0.5 M_{\odot}$, these stars should typically be relatively bright NIR sources. Therefore, all cases where an $\mathrm{X}$-ray source has an unexpectedly faint primary match and a considerably brighter secondary match, deserve special attention. We found four cases where the primary match was very faint ( $J \geq 18$, or $H \geq 17.5$, or $\left.K_{\mathrm{s}} \geq 17.2\right)$ and a brighter secondary match was present. For these cases, we replaced the original very faint primary match by this brighter secondary match.

Our final detailed inspection of the VISTA images revealed several cases, where an X-ray source for which the procedure match_xy.pro did not report a match, has a rather bright infrared source very close to the X-ray source position, but just outside the matching region. It is very likely that some of these cases are "false negative" rejections of physical matches. A quantitative estimate of the expected number of "false negative" rejections can be made as follows: In theory, the use of $99 \%$ probability boundaries for the match region should lead to about $1 \%$ false negative rejections of true matches, i.e., about 7 cases in our X-ray sample. In reality, this number will be somewhat higher, since the true positional errors will not perfectly

\footnotetext{
${ }^{12}$ The average surface density of VISTA catalog sources in the field of our Chandra pointing is 136 objects per square-arcminute. Since the typical $99 \%$ match region has a area of about 3 square-arcseconds, the expected probability of random associations with unrelated infrared sources is about $10 \%$.
}

follow a Gaussian distribution, but usually have somewhat wider "wings". This estimate agrees quite well with the number of 11 observed bright infrared sources that are located within $\leq 1.0^{\prime \prime}$ of the X-ray source position but just outside the formal matching region. We therefore decided to add these 11 cases to our cata$\log$ of infrared counterparts. In all other cases, where the angular distance between an X-ray source and a star in the VISTA images is larger than 1.0 ", we did not add matches "by hand", in order to keep the matching as objective as possible. Although relaxing the matching limits would yield a slightly higher rate of matches, it would also increase the number of unphysical chance associations. We prefer here a clean sample over a marginally larger, but less clean sample.

Finally, our visual inspection of the X-ray source positions on the VISTA images revealed 8 cases where a clear match is visually evident, but the infrared source was missing from the VISTA catalog. For these stars, we performed individual aperture photometry in the VISTA tile images and added them to our counterpart list.

In total and as the final result of our matching procedures, we have VISTA NIR counterparts for 500 of the 679 X-ray sources; the corresponding matching rate is $73.6 \%$. For 458 of these sources, VISTA photometry is available in all three bands. Table 3 lists the NIR magnitudes of the X-ray sources.

We note that these matching results are very well consistent with the expectation about the number of young stars $(\sim 500)$ and contaminants $(\sim 180)$ in the sample of X-ray sources as discussed in Sect. 2.6, since the population of contaminants should be dominated by extragalactic objects, and almost all X-ray detected AGN are expected to be so faint in the NIR regime $(J \gtrsim 20)$ that they should remain undetected in our VISTA images.

\subsection{Chandra - Spitzer matching results}

The matching of the X-ray source list with our Spitzer IRAC point source catalog with the match_xy procedure produced 272 successful primary matches. Since the positional uncertainties of mid-infrared sources in the Spitzer images are higher than those of the NIR sources in the VISTA images, the maximum allowed matching radii used for the Chandra - Spitzer matching are larger than those used for the Chandra - VISTA matching. Therefore, an additional step is necessary to make sure to get consistent Chandra - VISTA - Spitzer matches. For all reported Spitzer matches to Chandra sources we checked whether the Spitzer source position is consistent with a NIR source in the VISTA images. There were 14 cases, where a Spitzer source that was classified as a match to an X-ray source could be clearly identified with a VISTA sources that was not a match of the Chandra source. In these cases, we removed the Spitzer match.

The final detailed visual inspection of all X-ray source positions in the Spitzer images revealed ten sources where a clear infrared counterpart can be seen, but is missing from the Spitzer point source photometry catalog.

The whole identification procedure finally yields 268 Spitzer counterparts to the $679 \mathrm{X}$-ray sources; the corresponding matching rate is $39.5 \%$. For 215 of these sources photometry is available in the $3.6 \mu \mathrm{m}$ band, for 258 in the $4.5 \mu \mathrm{m}$ band, for 98 in the $5.6 \mu \mathrm{m}$ band, and for 46 in the $8.0 \mu \mathrm{m}$ band. The IRAC magnitudes of the X-ray sources are listed in Table 3. 


\subsection{Chandra - Herschel matching results}

Because of the relatively moderate angular resolution of the Herschel images and the often weak contrast between point-like sources and the generally strong surrounding cloud emission, the positional uncertainties for the Herschel point-like sources are considerably larger than those of the NIR and MIR sources. Therefore, we used a search radius of $5^{\prime \prime}$ to look for possible X-ray detections of Herschel sources.

Five X-ray sources are located within $5^{\prime \prime}$ of a Herschel source. In all five cases, the X-ray source has a clear match with a NIR point source visible in the VISTA images. Therefore, these objects are no pre-stellar or very young protostellar (class 0 ) objects, but more evolved YSOs of class I or class II.

\subsection{X-ray and infrared sources in the clusters NGC 3324 and G286.38-0.26}

The stellar cluster NGC 3324 harbors the highest and densest concentration of X-ray sources (see Fig. 5). It contains $82 \mathrm{X}$-ray sources in a $2.5^{\prime} \times 2.5^{\prime}$ region. Among these are the three known O-type stars in the Gum 31 region. The VISTA image shows several hundred faint stars, many of which are probably low-mass cluster members with X-ray luminosities below our detection limit. A more detailed discussion of the X-ray detected population will be given in Sect. 6. Neither the VISTA or Spitzer, nor the Herschel images show significant amounts of cloud emission in or around this cluster. This implies that no active star formation is going on in this region, and the young stars have already largely dispersed their natal clouds.

The stellar cluster G286.38-0.26 is the second most significant spatial concentration of young stars in the area. We find 42 $\mathrm{X}$-ray sources in a $3.8^{\prime} \times 2^{\prime}$ region. In contrast to NGC 3324 , this cluster is still associated to large amounts of cloud material, which is prominently seen in the Herschel and Spitzer images and is traced by the diffuse nebulosity seen in the above VISTA image. The cloud column-densities derived from the Herschel data for this region (see Preibisch et al. 2012; Ohlendorf et al. 2013) ranges from about $N_{\mathrm{H}} \approx 1 \times 10^{22} \mathrm{~cm}^{-2}\left(A_{V} \approx 5 \mathrm{mag}\right)$ to $N_{\mathrm{H}} \approx 4 \times 10^{22} \mathrm{~cm}^{-2}$ ( $\left.A_{V} \approx 20 \mathrm{mag}\right)$. Embedded in these clouds are several bright protostellar infrared sources (some of which are discussed in the next section). However, there is also a number of optically visible stars which show rather low extinction and no infrared excesses. Thus, the cluster contains a mix of very young objects, ongoing star formation activity, and more evolved, older stars. A multi-wavelength image comparison of this cluster can be seen in Fig. 12 in Ohlendorf et al. (2013).

\section{X-ray properties of the brightest infrared and optical objects}

The properties of the particularly interesting objects, that warrant a detailed look, are described in this section.

\subsection{Embedded infrared sources}

J103801.84-584642.4: this source is identified with the infrared source MSX6C G286.3747-00.2630 in the cluster G286.38-0.26. This object is very faint in optical images, very bright in the NIR, and one of the brightest mid-infrared sources in this area (especially in the $24 \mu \mathrm{m}$ MIPS image). These properties suggest it to be a very young, embedded YSO with large amounts of circumstellar material. The X-ray lightcurve shows no strong variability. The emission is relatively hard (median photon energy $2.33 \mathrm{keV}$ ), and XPHOT estimates a column density of $N_{\mathrm{H}} \sim 1.5 \times 10^{22} \mathrm{~cm}^{-2}$ (corresponding to a visual extinction of $\left.A_{V} \sim 8 \mathrm{mag}\right)$. The X-ray luminosity estimated with XPHOT is $\log \left(L_{\mathrm{X}}[\mathrm{erg} / \mathrm{s}]\right) \approx 31.4$. According to the general relation between X-ray luminosity and stellar mass for young stellar objects derived in the Chandra Orion Ultradeep Project (see Preibisch et al. 2005), steady X-ray emission at such a level suggest a stellar mass in the range of $\sim 2-3 M_{\odot}$.

J103800.40-584643.3: this is an optically invisible source in the G286.38-0.26 cluster. It is invisible in our VISTA $J$ - and $H$-band images (implying $J \gtrsim 21, H \gtrsim 20$ ), but appears as a faint $K_{\mathrm{s}}$-band source with $K_{\mathrm{s}} \sim 16$. It is quite bright in the IRAC images, but undetected in the MIPS image. The Herschel far-infrared maps show a dense cloud clump with a hydrogen column density of $N_{\mathrm{H}} \approx 3 \times 10^{22} \mathrm{~cm}^{-2}\left(A_{V} \approx 15 \mathrm{mag}\right)$ near its position, suggesting that this source is a particularly deeply embedded YSO. This source showed a strong flare during our Chandra observation and has a very hard median photon energy of $5.4 \mathrm{keV}$. XPHOT estimates a very high column density of $N_{\mathrm{H}} \sim 4 \times 10^{23} \mathrm{~cm}^{-2}$ (corresponding to a visual extinction of $A_{V} \sim 200 \mathrm{mag}$ ) and a very high X-ray luminosity of $\log \left(L_{\mathrm{X}}[\mathrm{erg} / \mathrm{s}]\right) \approx 32.4$ for this object.

J103806.57-584000.4: this Chandra source corresponds to an optically invisible infrared source with very red NIR colors ( $\left.J-H=2.92, H-K_{\mathrm{s}}=1.60\right)$, which is located at the tip of a prominent pillar at the eastern edge of the $\mathrm{H}$ II region. An image of this object and the pillar and the near- to far-infrared spectral energy distribution can be seen in Ohlendorf et al. (2013), where it is listed as J103806.6-584002. It is classified as a class I object with a stellar mass of $M_{*} \approx 1.7 M_{\odot}$ that is surrounded by a circumstellar disk and a massive envelope and has a total luminosity of $L \approx 40 L_{\odot}$. The XPHOT estimate of the column density of $N_{\mathrm{H}} \sim 2 \times 10^{22} \mathrm{~cm}^{-2}$ (corresponding to a visual extinction of $A_{V} \sim 10 \mathrm{mag}$ ) is consistent with these infrared results. The XPHOT estimate of its X-ray luminosity is $\log \left(L_{\mathrm{X}}[\mathrm{erg} / \mathrm{s}]\right) \approx 30.7$, suggesting a fractional X-ray luminosity of $\log \left(L_{\mathrm{X}} / L_{\mathrm{bol}}\right) \approx-2.9$.

\subsection{Optically bright stars}

\subsubsection{The early-type $(O, B, A)$ stars}

The three known O-type stars in Gum 31 are the components A, $\mathrm{B}$, and C of HD 92 206, located in the central cluster NGC 3324. They are the most luminous and massive stars in the region. All three are clearly detected as X-ray sources, and our analysis reveals two additional, yet unreported components.

HD 92206 A: the optically brightest component HD 92206 A is of spectral type O6.5 V. It can be clearly identified with the brightest X-ray source (J103722.27-583723.0) in our Chandra observation. The 920 source counts allow a rather detailed analysis of the X-ray spectrum. The fit with a single-temperature spectral model (Fig. 6, top) leaves systematic excesses in the soft as well the hard parts of the spectrum. Although a twotemperature spectral model yields a statistically acceptable fit with temperature components of $k T_{1}=0.38 \pm 0.1 \mathrm{keV}$ and $k T_{2}=2.32 \pm 1.14 \mathrm{keV}$, there is still a systematic excess in the observed spectrum at high photon energies (see Fig. 6). We 

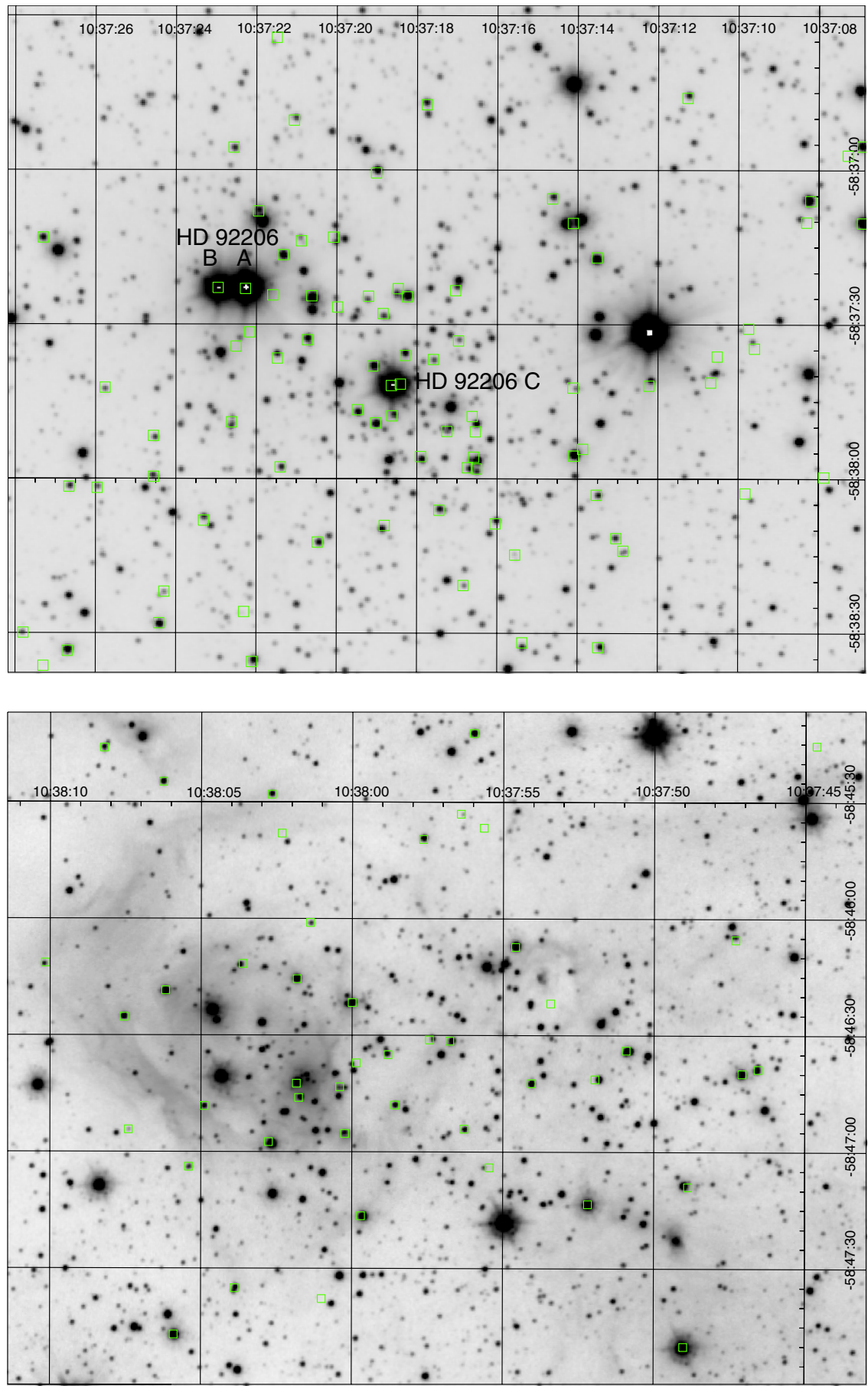

Fig. 5. Top: negative representation of the VISTA $H$-band image of the cluster NGC 3324 (top) and the VISTA $K_{\mathrm{s}}$-band image of the cluster G286.38-0.26 (bottom). The positions of the X-ray sources are shown by the green boxes. We note that the $\mathrm{X}$-ray source position uncertainties are in most cases considerably smaller than the size of the box symbols; the rather large $2^{\prime \prime}$ box size was chosen for a clarity of display. therefore considered a three-temperature model, which yields a better fit with temperature components of $k T_{1}=0.20 \pm 0.08 \mathrm{keV}$, $k T_{2}=0.54 \pm 0.54 \mathrm{keV}$, and $k T_{3}=5.17 \pm 5.17 \mathrm{keV}$. Although the temperatures are not very tightly constrained, this model reproduces the shape of the spectrum very well (see Fig. 6). The ratio of the corresponding emission measures is 60.2:24.9:3.21, clearly showing that the X-ray spectrum is dominated by rather soft emission with plasma temperatures of $\$ 6 \times 10^{6} \mathrm{~K}$, as typical for O-type stars. The X-ray luminosity of $\log \left(L_{\mathrm{X}}[\mathrm{erg} / \mathrm{s}]\right)=$ $31.88 \pm 0.24$ is also in the typical range for late O-type stars. Assuming that the bolometric luminosity of HD $92206 \mathrm{~A}$ is $\log \left(L_{\mathrm{bol}} / L_{\odot}\right)=5.23$ according to its spectral type and the models of Martins et al. (2005), we find a fractional X-ray luminosity of $\log \left(L_{\mathrm{X}} / L_{\mathrm{bol}}\right) \approx-6.9$. This is very close to the canonical value of fractional X-ray luminosities for O-type stars in general and also in the Carina nebula: Gagné et al. (2011) found that the great majority of $\mathrm{O}$ stars in the Carina nebula have $\log \left(L_{\mathrm{X}} / L_{\mathrm{bol}}\right)$ ratios between -6.8 and -8.0 , with a mean value of -7.23 .

The hard $5.17 \mathrm{keV}$ component (corresponding to a plasma temperature of about $60 \times 10^{6} \mathrm{~K}$ ), is however, quite unusual for an O-type star. One possibility is that this unexpected hard $\mathrm{X}$-ray emission originates not from the O-star but from another nearby object, such as a coronally active late type companion. We therefore performed a detailed inspection of the available optical and infrared images of HD 92206 A to explore this possibility. An archival HST image, obtained with the WFC camera of ACS in the filter F658N, is shown in Fig. 7. This image shows a faint point source at the position 

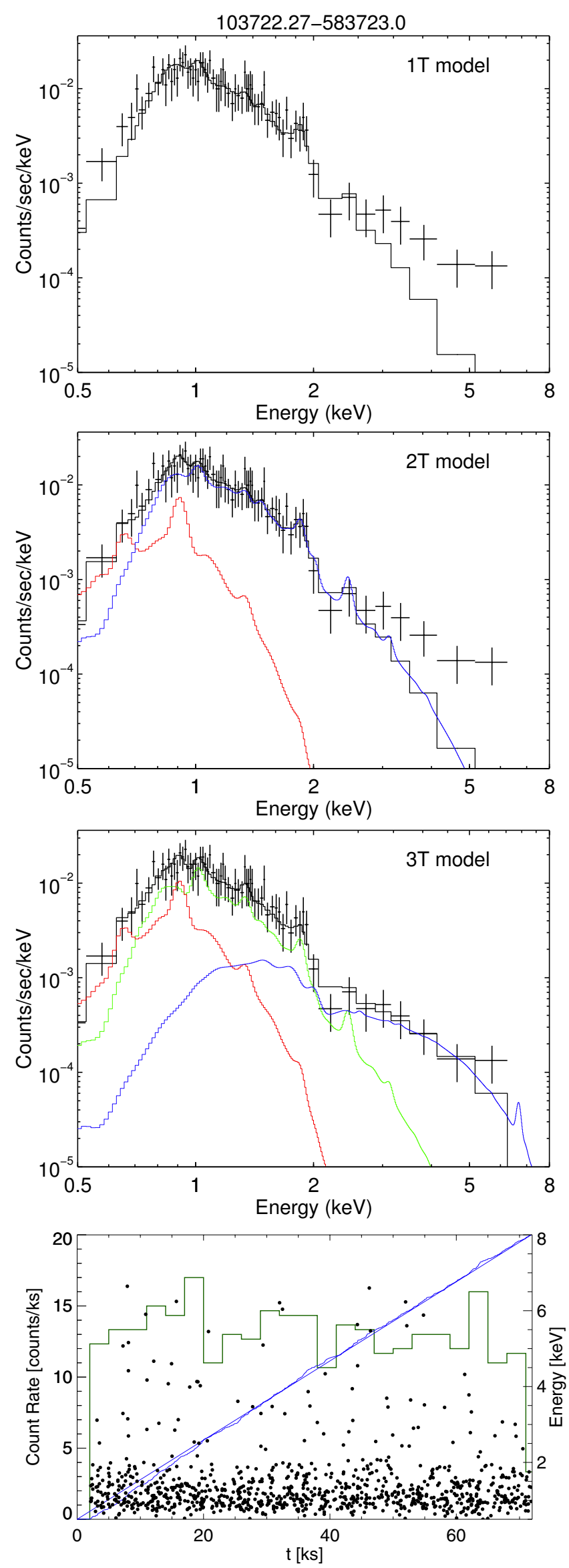

Fig. 6. X-ray spectral fits and lightcurve of the O6.5 star HD 92206 A.

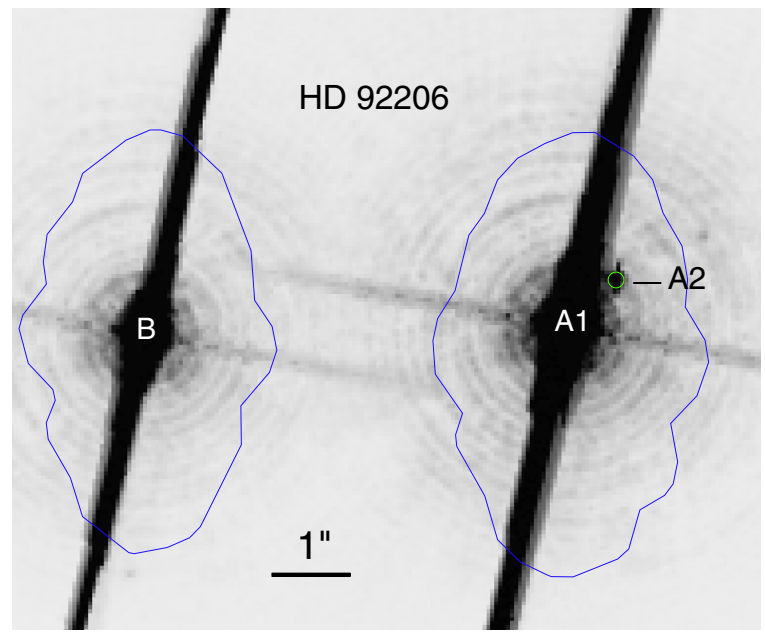

Fig. 7. Optical Hubble Space Telescope image of HD 92206 A and B in the filter $F 658 \mathrm{~N}$; north is up and east is to the left. The blue polygons show the X-ray extraction regions, which enclose $90 \%$ of the PSF (note that the Chandra PSF is elongated and enlarged at the rather large offaxis angle $\left(4.3^{\prime}\right)$ of the object). The faint companion of HD $92206 \mathrm{~A}$ can be seen about $0.8^{\prime \prime}$ northwest and is marked as "A2".

$\mathrm{RA}=10^{\mathrm{h}} 37^{\mathrm{m}} 22.206^{\mathrm{s}}$, Dec $=-58^{\circ} 37^{\prime} 22.26^{\prime \prime}$, i.e., at an angular offset of $0.83^{\prime \prime}$ from the O-star, corresponding to a projected physical separation of $\approx 1900 \mathrm{AU}$. We will designate the companion as HD 92206 A2, and the primary star as HD 92206 A1. As can be seen in Fig. 7, the X-ray source position is in very good agreement with the location of the bright O-star primary HD 92206 A1, clearly showing that most of the observed X-ray emission must predominantly come from HD 92206 A1, not from the companion A2. However, the location of the companion is close enough that its X-ray photons are clearly included in the extraction region that is centered on HD 92206 A1.

In order to obtain an estimate of the stellar properties of the companion, we performed aperture photometry in another HST ACS/WFC image that was obtained in the medium-band filter F550M (unfortunately, not data obtained through broadband filters are available). This yielded a magnitude of $m_{F 550 M} \approx$ $18.1 \pm 0.5$, where the rather large uncertainty is dominated by the choice of the sky region, since the object is located in the outer parts of the point-spread-function of the much brighter star HD 92206 A1. According to the PMS stellar models of Siess et al. (2000), the derived magnitude is consistent with the assumption that the companion A2 is a young ( 1 Myr old) star with a mass of about $0.5-1 M_{\odot}$, if the extinction is not much higher than $A_{V} \lesssim 3 \mathrm{mag}$. The companion could thus well be a young late-type star, which produces coronal X-ray emission, what might explain the excess of hard X-ray emission seen in the spectrum of HD 92206 A. We note that the X-ray lightcurve shows no significant variability; therefore, the hard X-ray emission of the system HD $92206 \mathrm{~A} 1 / \mathrm{A} 2$ is not related to coronal flaring activity.

HD 92206 B (=CD -573380 B; spectral type O6.5 V) corresponds to X-ray source J03722.95-583722.9 and yielded 226 source counts in our Chandra observation. A fit to the X-ray spectrum with a two-temperature model yields plasma components with $k T_{1}=0.22 \pm 0.05 \mathrm{keV}$ and $k T_{2}=0.64 \pm 0.05 \mathrm{keV}$ and gives an X-ray luminosity of $\log \left(L_{X}[\mathrm{erg} / \mathrm{s}]\right)=31.20 \pm 0.15$. Assuming again a bolometric luminosity of $\log \left(L_{\text {bol }} / L_{\odot}\right)=5.23$ 


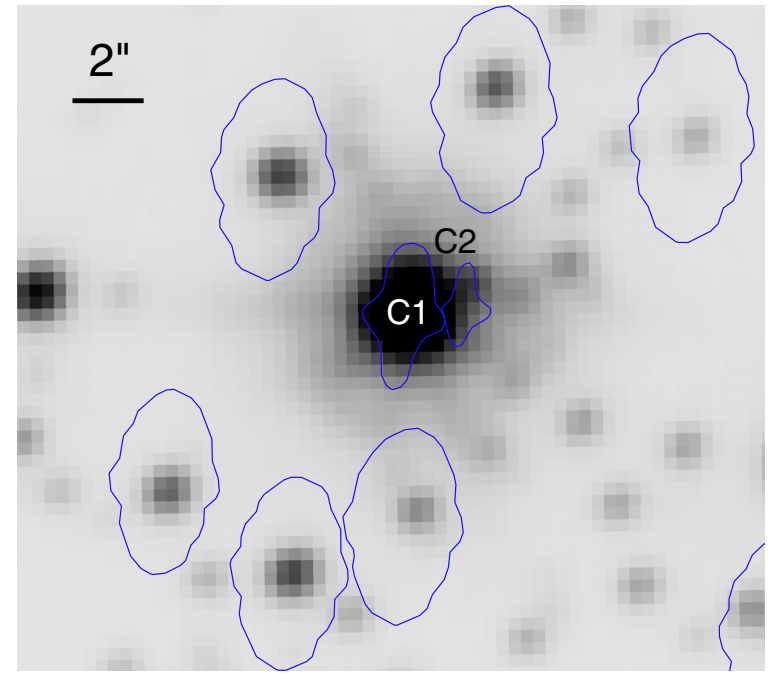

Fig. 8. VISTA $K_{\mathrm{s}}$-band image of the region around HD $92206 \mathrm{C}$; north is up and east is to the left. The blue polygons show the individual $\mathrm{X}$-ray extraction regions. The faint companion of HD $92206 \mathrm{C}$ can be seen about 1.7" northwest and is marked as " $\mathrm{C} 2$ ". The extraction region for the source $\mathrm{C} 2$ was automatically shrunken to avoid overlap with the extraction region of source $\mathrm{C} 1$, and thus encloses only $40 \%$ of the PSF. We note that the Chandra PSF is elongated and enlarged at the rather large off-axis angle $\left(4.3^{\prime}\right)$ of theses objects.

according to its spectral type and the models of Martins et al. (2005), we find a fractional X-ray luminosity of $\log \left(L_{X} / L_{\text {bol }}\right) \approx$ -7.6 for this star. The lightcurve shows no significant variability. These properties are in the typical range as reported for the O-type stars in the Carina nebula by Gagné et al. (2011).

HD $92206 \mathrm{C}(=\mathrm{CD}-573378)$ is the third known O-type star in NGC 3324. A spectral type of $08.5 \mathrm{Vp}$ is listed in the SIMBAD catalog. However, the spectroscopic monitoring study of Campillay et al. (2007) found HD $92206 \mathrm{C}$ to be a doublelined spectroscopic binary with a period of 2.02 days. They derived spectral types of $\mathrm{O} 7.5 \mathrm{~V}+\mathrm{B} 0 \mathrm{~V}$ for the two components and determined a mass-ratio of about 0.7 from the radial velocity curve analysis.

HD $92206 \mathrm{C}$ is clearly detected by Chandra as source J103718.63-583741.9. It yielded 119 source counts, and our spectral fit gives a plasma temperature of $k T=0.57 \pm 0.14 \mathrm{keV}$ and an X-ray luminosity of $\log \left(L_{\mathrm{X}}[\mathrm{erg} / \mathrm{s}]\right)=30.98 \pm 0.29$. Assuming a bolometric luminosity of $\log \left(L_{\mathrm{bol}} / L_{\odot}\right)=5.05$ (according to the 07.5 spectral type of the primary and the models of Martins et al. 2005), we find a fractional X-ray luminosity of $\log \left(L_{\mathrm{X}} / L_{\mathrm{bol}}\right) \approx-7.3$ for this star. The lightcurve shows at most marginal indications for variability. These values are again in the typical range observed for late O-type stars.

Interestingly, the Chandra image revealed another X-ray source, J103718.41-583741.6, at an angular separation of just 1.7" from HD 92206 C. Our detailed inspection of the VISTA images reveals a marginally resolved companion near the position of the second X-ray source and thus confirms the presence of two distinct sources (see Fig. 8). We designate the secondary component as HD 92206 C2. Unfortunately, the small angular separation from the very bright O-type primary star $\mathrm{C} 1$ did not allow us to obtain reliable photometric flux estimates from the VISTA data. All we can say is that the companion is considerably fainter in the NIR than the main component. In the available
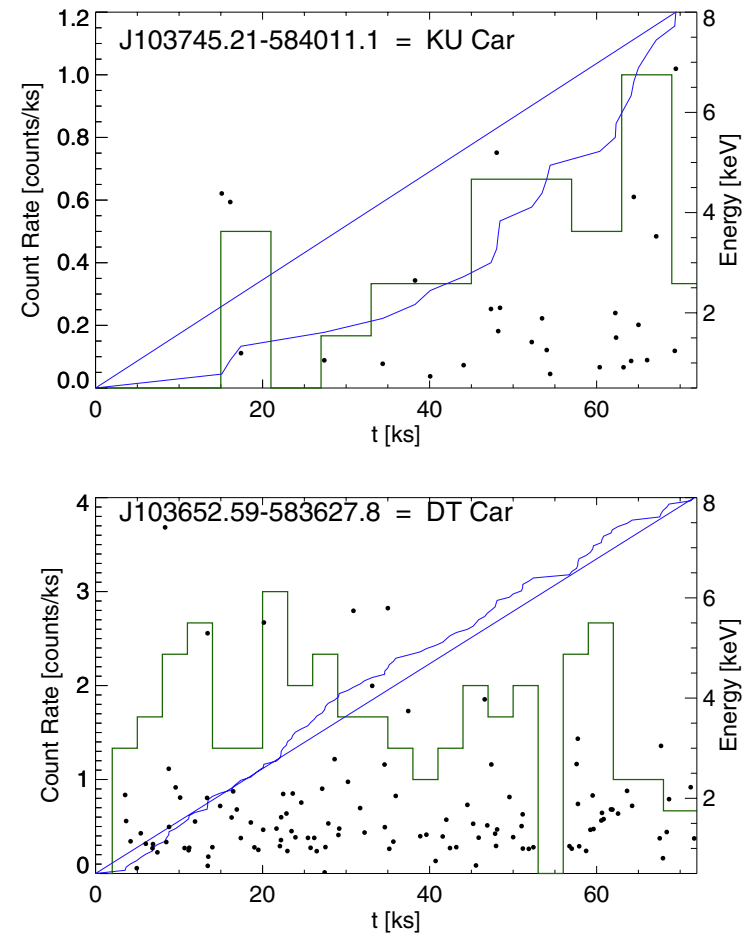

Fig. 9. Chandra X-ray lightcurves of the two Algol stars KU Car (J103745.21-584011.1) and DT Car (J103652.59-583627.8).

HST images, this stellar system is located just outside the fieldof-view and can thus not be analyzed.

The extraction of the X-ray source J103718.41-583741.6 had to be done with a reduced aperture size in order to avoid overlap with the nearby source J103718.63-583741.9 (=HD 92206 C); the extraction region contains only $40 \%$ of the PSF and yielded just 16 net counts, too few for a detailed spectral analysis. The median photon energy is $1.19 \mathrm{keV}$, and the estimated X-ray luminosity derived with XPHOT is $\log \left(L_{X}[\mathrm{erg} / \mathrm{s}]\right) \approx 30.57$. The fact that the companion is considerably less bright in the X-ray as well as in the NIR regime, suggests that it is probably a late-type star.

The known B- and A-type stars in Gum 31, HD 303080 (spectral type B), HD 92145 (spectral type A2), HD 92207 (spectral type A0Iae), and HD 303094 (spectral type A2), are not detected as X-ray sources. This is not surprising, since no X-ray emission is expected from stars in the spectral range $\sim \mathrm{B} 2$ to $\sim \mathrm{A} 9$ (see, e.g., discussion in Stelzer et al. 2005).

\subsubsection{The Algol-type eclipsing binaries KU Car and DT Car}

These two objects are detected as X-ray sources and here we provide a brief discussion of their X-ray properties.

KU Car is identified with the X-ray source J103745.21584011.1. The $27 \mathrm{X}$-ray source counts are not enough for spectral fitting, but we note that the X-ray luminosity estimated with $\mathrm{XPHOT} \mathrm{is} \log \left(L_{\mathrm{X}}[\mathrm{erg} / \mathrm{s}]\right) \approx 31.0$, if we assume a distance of $2.3 \mathrm{kpc}$. The X-ray lightcurve of this source shows clear evidence of a substantial increase in the count rate during the $\approx 19 \mathrm{~h}$ of our observation. 

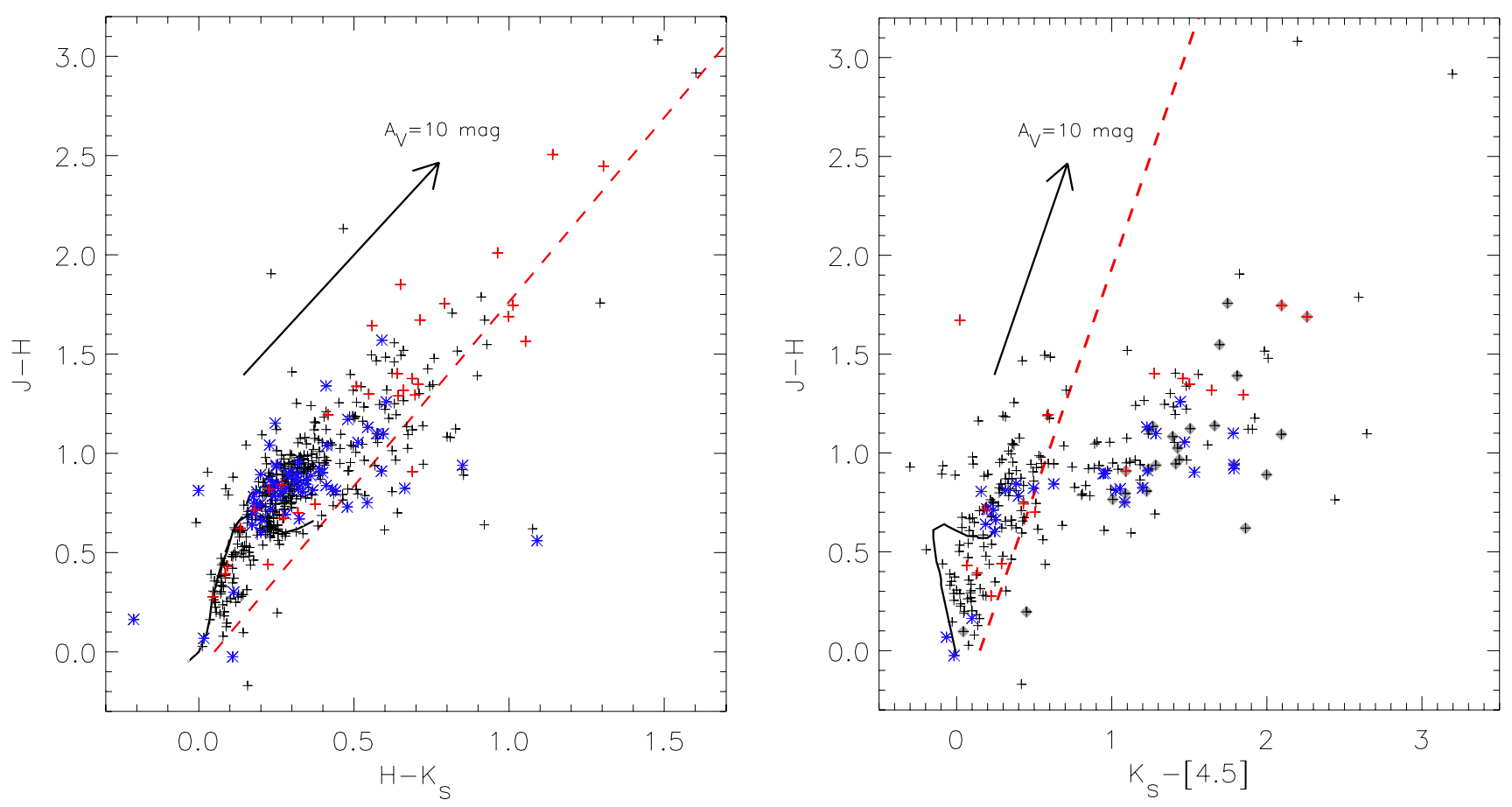

Fig. 10. Left: NIR $J-H$ versus $H-K$ color-color diagram of all X-ray selected sources (crosses). The X-ray sources in the NGC 3324 cluster region are marked by blue asterisks, whereas the sources in the G286.38-0.26 cluster region are marked by red crosses. The solid line shows the main sequence, the arrow shows a $A_{V}=10$ mag reddening vector with slope 1.85 , and the red dashed line marks the separation between the photospheric reddening band and the infrared excess region. Objects are classified as NIR excess sources if they lie at least 0.05 mag to the right of the reddening band and above $J-H=0$. Right: near- to mid-infrared $J-H$ versus $K-$ [4.5] color-color diagram of all X-ray selected sources. The symbols have the same meaning as in the left plot. Sources with NIR excesses are additionally marked by filled grey diamonds.

It is interesting to compare the $\mathrm{X}$-ray lightcurve to the optical lightcurve of this system, for which O'Connell (1956) determined a period of $5.921121 \pm 0.000007$ days. According to the lightcurve elements derived in that study (where the phase $\phi=0$ corresponds to the minimum I), the phase at the start of our Chandra observation is $\phi=0.54$, and the phase at the end of our Chandra observation is $\phi=0.68$. The minimum II (phase $\phi=0.5$ ) happened $5.88 \mathrm{~h}$ before start of the Chandra observation. According to the optical lightcurve shown in Fig. 5 of O'Connell (1956), the visual brightness of the system increases within that period (i.e., it includes the final part of the occultation). The rise of the X-ray count rate during our Chandra observation is qualitatively consistent with this and suggests that the low count rate during the first hours of the observation are due to a (partial) X-ray eclipse in the system.

DT Car, the second known Algol-type eclipsing binary in Gum 31, has a period of 4.2866 days and a spectral type of A2:+[G6Iv] as listed in SIMBAD. This object is located just at the rim of the Gum $31 \mathrm{H}$ II region. It is detected as X-ray source J103652.59-583627.8 in our Chandra data with 117 source counts. The X-ray luminosity derived from our spectral fit is $\log \left(L_{\mathrm{X}}[\mathrm{erg} / \mathrm{s}]\right)=31.06 \pm 0.16$ (assuming a distance of $2.3 \mathrm{kpc}$ ).

According to the lightcurve elements listed in the VizieR catalog, the range of phases covered by our Chandra observation is from $\phi=0.45$ to $\phi=0.65$. This means that the minimum II $(\phi=0.5)$ should have occurred $17.2 \mathrm{ks}$ after the start of the observation. However, no indication of variability is seen in the $\mathrm{X}$-ray lightcurve at this time. This may be related to uncertainties in the optical lightcurve elements; no error bars are given for the period or the epoch of $\phi=0$ in the VizieR catalog, but we note that changing the last given digit of the period by 1 is enough to shift the epoch of phase $\phi=0.5$ outside the range of times covered by our Chandra observation.

\section{Properties of the X-ray selected young stellar population}

\subsection{Infrared excesses of the X-ray sources}

A quantification of the fraction of stars that are still surrounded by circumstellar material (disks) can provide important information about the evolutionary state of a region. A good way to do this is to look for excesses in near- or mid-infrared color-color diagrams.

In Fig. 10 we show a NIR $J-H$ vs. $H-K_{\mathrm{s}}$ color-color diagram for the X-ray selected sources. Objects in this diagram are classified as NIR excess sources if they lie at least $0.05 \mathrm{mag}$ to the right of the reddening line, which is based on the intrinsic colors of dwarfs (Bessel \& Brett 1988) and an extinction vector with slope 1.85 . In this way, 26 of the $472 \mathrm{X}$-ray sources with complete $J, H$, and $K_{\mathrm{s}}$ photometry are classified as NIR excess sources, i.e., we find a NIR excess fraction of $5.5 \%$ in the X-ray selected population.

It is well known that NIR emission only traces the hottest dust, close to the inner edge of the disk. Therefore, the NIR excesses diminish rather quickly during the first few million years of disk evolution. Excesses in the mid-infrared range, which are caused by somewhat cooler disk material, are known to persist considerably longer. We have therefore also constructed a color-color-diagram including the mid-infrared IRAC2 $4.5 \mu \mathrm{m}$ band. As expected, the near/mid-infrared $J-H$ vs. $K_{\mathrm{s}}-[4.5]$ color-color diagram yields a considerably higher excess fraction 

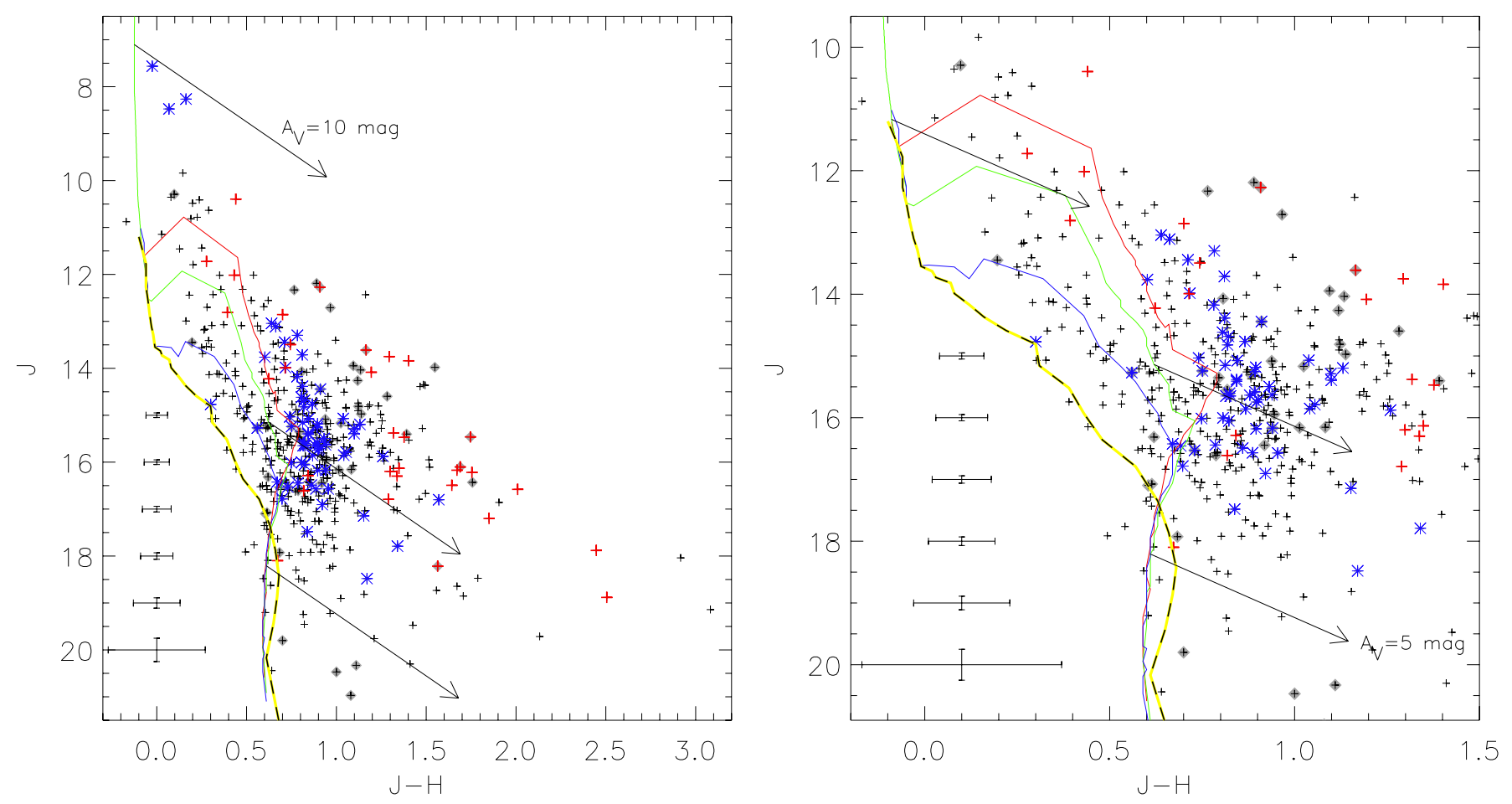

Fig. 11. Left: NIR color-magnitude diagram of the X-ray selected sources (crosses). The X-ray sources in the NGC 3324 cluster region are marked by blue asterisks, whereas the sources in the G286.38-0.26 cluster region are marked by red crosses. Sources with NIR excesses are additionally marked by filled grey diamonds. The solid lines show isochrones for ages of $1 \mathrm{Myr}$ (red), $3 \mathrm{Myr}$ (green), and $10 \mathrm{Myr}$ (blue) composed from the models of Baraffe et al. (1998) for the mass range 0.02 to $0.5 M_{\odot}$, Siess et al. (2000) for the mass range 0.5 to $7 M_{\odot}$, and Lejeune \& Schaerer (2001) (model iso-c020-0650) for the mass range 7 to $70 M_{\odot}$; the dashed line shows the ZAMS from Siess et al. (2000). The arrows indicate reddening vectors for $A_{V}=10 \mathrm{mag}$ starting at the location of $3 \mathrm{Myr}$ old stars with masses of $35 M_{\odot}, 1 M_{\odot}$, and $0.1 M_{\odot}$. The row of error-crosses in the lower left part of the plot shows the typical magnitude-dependent photometric uncertainties. Right: zoom into the central region of the CMD. The arrows indicate reddening vectors for $A_{V}=5$ mag starting at the location of 3 Myr old stars with masses of $7 M_{\odot}, 1 M_{\odot}$, and $0.1 M_{\odot}$.

of $40.9 \%$ (101 of the 247 objects with complete photometry in the $J, H, K_{s}$, and [4.5] bands).

The NIR color-color diagram also allows us to characterize the extinction of the X-ray selected stars. Most objects show extinctions of about $2 \mathrm{mag}$, while roughly $10 \%$ of the sample have values of $A_{V} \gtrsim 5 \mathrm{mag}$, with extreme values around $A_{V} \sim 20 \mathrm{mag}$.

\subsection{Color-magnitude diagram of the $X$-ray sources}

Color-magnitude diagrams (CMDs) can be used to derive information about stellar ages and masses. However, several factors, such as differential extinction, infrared excesses, unresolved binary companions, and photometric variability, can change the position of individual stars in the diagram and thus strongly adulterate the derived stellar age and mass estimates. The corresponding fundamental limitations of age and mass determinations based on the analysis of CMDs are discussed in Preibisch et al. (2011c) and also (in more detail) in Preibisch (2012). Nevertheless, it is possible to estimate the typical ages for the different stellar populations in Gum 31.

The NIR CMD of the X-ray selected objects in Gum 31 is shown in Fig. 11. The bulk of the X-ray stars have masses in the range $\sim 0.5 M_{\odot}$ to $\sim 3 M_{\odot}$. Below $\sim 0.5 M_{\odot}$, the number drops strongly, in good agreement with the expectation from the X-ray detection limit. Just a few objects are seen at locations corresponding to masses around $\sim 0.1 M_{\odot}$.

The positions of the three O-type stars suggest moderate extinctions of $A_{V} \sim 2$ mag for these objects; this agrees very well with the column densities derived from the fits to the X-ray spectra of HD $92206 \mathrm{~A}$ and B $\left(N_{\mathrm{H}} \approx 4 \times 10^{21} \mathrm{~cm}^{-2}\right.$, corresponding to $A_{V} \approx 2$ mag for both objects).

\subsection{CCD and CMD results for the cluster NGC 3324}

The CCD suggests extinctions of typically $A_{V} \sim 2$ mag for the $\mathrm{X}$-ray selected objects in this cluster. The NIR excess fraction is $9.5 \%$ (6 out of 63 objects with complete photometry in the $J$, $H$, and $K_{\mathrm{s}}$ bands), while the MIR excess fraction is $51.7 \%$ (15 out of 29 objects with complete $J, H, K_{\mathrm{s}}$, and [4.5] photometry).

In the CMD, almost all stars in NGC 3324 are located to the right of the $1 \mathrm{Myr}$ isochrone. There are only two exceptions: one is source J103721.95-583708.0, that appears close to the $10 \mathrm{Myr}$ isochrone, the other one is J103714.63-583705.5 that appears at the ZAMS. We note that J103721.95-583708.0 shows a clear NIR excess, and thus its position in the CMD can not be used to infer an age. This leaves J103714.63-583705.5 as the only object in the cluster area that appears clearly offset (in the CMD) from the rest of the cluster population; this suggests that this object may not be a member of this cluster, but is only seen there due to projection effects. For example, it could be a reddened background O-type star.

Taking the typical reddening of $A_{V} \sim 2$ mag into account, these locations of the stars in the CMD suggest an age of $\lesssim 2 \mathrm{Myr}$. The fact that the cluster region is free from any dense clouds, i.e., that the cluster stars must have already completely dispersed their natal cloud, implies that the age should be not much less that $\gtrsim 1$ Myr. 
The NIR excess fraction of $9.5 \%$ and the MIR excess rate of $51.5 \%$ are consistent with an age of $\sim 2$ Myr (see, e.g., Fedele et al. 2010).

Therefore, our final age estimate for NGC 3324 is $\approx 1-2 \mathrm{Myr}$. We note that this value agrees well to the expansion age of Gum 31 HII region, which has been estimated to be about 1.5 Myr (Ohlendorf et al. 2013).

\subsection{Results for the cluster G286.38-0.26}

The CCD shows a roughly bimodal distribution of extinctions: several stars have rather low extinction values $\left(A_{V} \lesssim 1 \mathrm{mag}\right)$, whereas several others show substantial extinction of $A_{V} \gtrsim$ 5 mag. The NIR excess fraction is $16.1 \%$ (5 out of 31 objects with complete photometry in the $J, H$, and $K_{\mathrm{s}}$ bands), while the MIR excess fraction is $52.9 \%$ (9 out of 17 objects with complete $J, H, K_{\mathrm{s}}$, and [4.5] photometry).

All but three of the stars in the cluster area are located to the right of the $1 \mathrm{Myr}$ isochrone in the CMD. These three apparently older stars are in the upper part of the CMD, which indicates ages of about 2-3 Myr; these objects belong to the group of bright optically visible stars in the region.

All other X-ray sources in this region appear to have ages of $\lesssim 1$ Myr and most of them show substantial amounts of extinction. Since there are large amounts of dense cloud material left, in which several deeply embedded young stellar objects are found, star formation is clearly going on in this cluster. Our final final age range estimate for G286.38-0.26 is therefore \1-2 Myr. The NIR excess fraction of $16 \%$ and MIR excess fraction of $53 \%$ is consistent with this.

\subsection{The distributed population}

We finally discuss the population of X-ray selected stars in the H II region that is not located in the clusters NGC 3324 and G286.38-0.26.

The CMD of the distributed stars is clearly different from the CMDs of the clusters NGC 3324 and G286.38-0.26 because it shows a broader spread of colors. The considerable number of objects to the left of the $3 \mathrm{Myr}$ isochrone and the presence of several objects near the $10 \mathrm{Myr}$ isochrone suggest a rather broad age distribution, with ages of up to $\sim 10 \mathrm{Myr}$ for this population. The excess rates of only 4\% (NIR) and 38\% (MIR) also suggest older ages for at least some part of this population.

\section{Global properties of the young stellar population in Gum 31}

\subsection{The size of the $X$-ray selected population}

In the study of Feigelson et al. (2011), the observed distribution of X-ray luminosities (the so-called "X-ray luminosity function", $X L F$ ) of the sources in different parts of the Carina nebula was used to derive estimates of the underlying total stellar populations by comparison to the XLF of the Orion nebula cluster (ONC), which was particularly well studied in X-rays in the context of the Chandra Orion Ultradeep Project (COUP; see Getman et al. 2005).

We use a similar strategy here to obtain information about the total population of young stars in the Gum 31 area. In Fig. 12 (upper panel) we compare the distribution of X-ray luminosity estimates (see Sect. 2.4) for our Gum 31 sample to the COUP data. Both distributions show a qualitatively similar increase in the source numbers when going from the highest towards
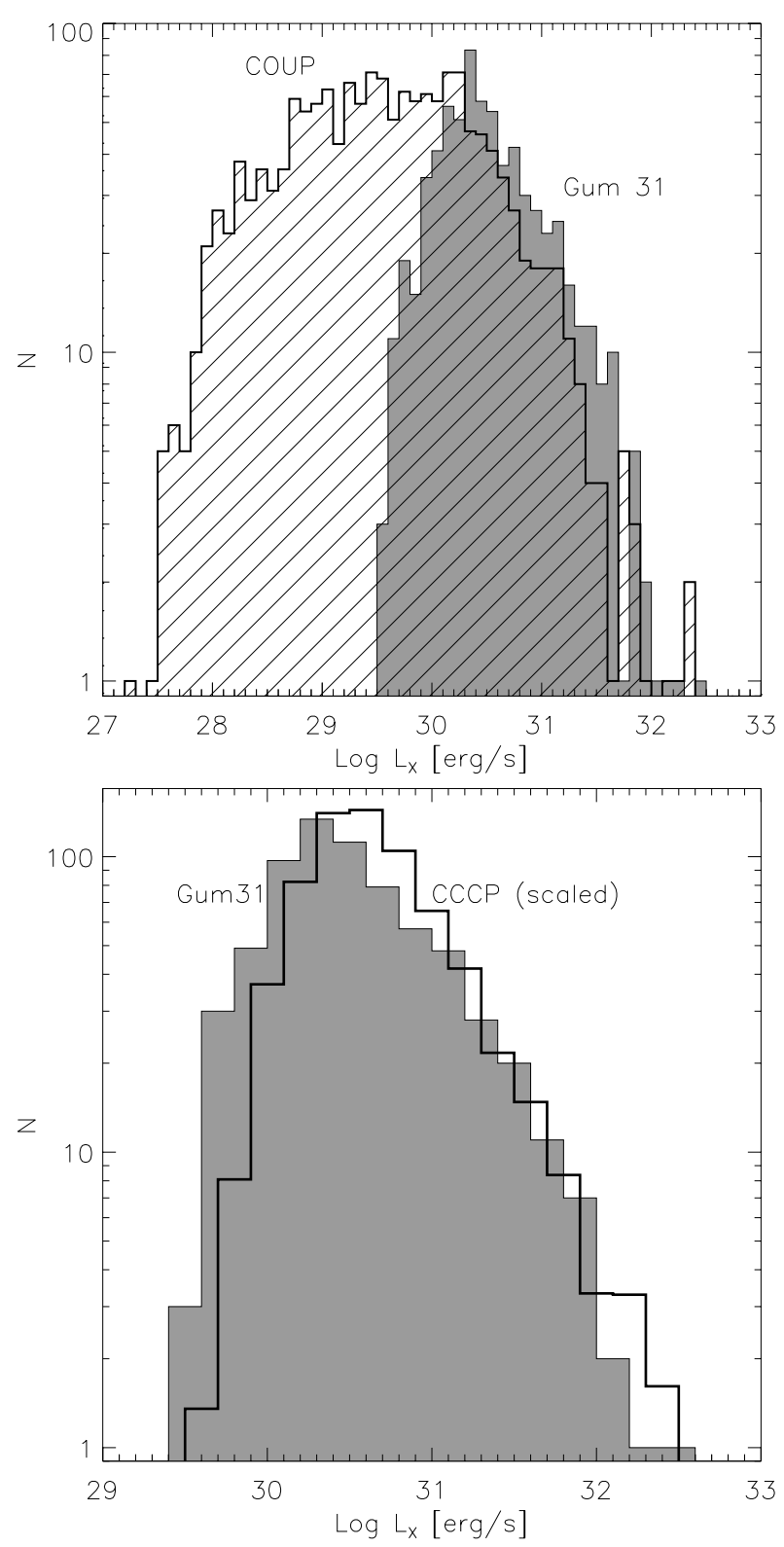

Fig. 12. Comparison of the X-ray luminosity function of the Chandra sources in our Gum 31 observation (grey-shaded histogram) to (top) the XLF of the Orion nebula cluster (dashed histogram) (from the COUP data; Getman et al. 2005), and to (bottom) the XLF for the CCCP sources as shown in Fig. 3 of Feigelson et al. (2011), scaled to account for the different sample sizes.

lower X-ray luminosities. As a consequence of the larger target distance and the shorter exposure time, the Gum 31 distribution turns over and drops down at a much higher luminosities $\left(\log \left(L_{\mathrm{X}}[\mathrm{erg} / \mathrm{s}]\right) \approx 30.3\right)$ than does the COUP data. Above this limit, however, the ratio of the number of X-ray sources should be approximately equal the ratio of the respective population sizes.

For the Gum 31 sample, we count $445 \mathrm{X}$-ray sources in the luminosity range $\log \left(L_{\mathrm{X}}[\mathrm{erg} / \mathrm{s}]\right)=[30.3 \ldots 32.0]$, whereas the COUP sample has 305 objects in this range. Therefore, the number of X-ray emitting stars in Gum 31 is about 1.46 times higher than in the ONC. If we assume the ONC population to consist of 2800 stars (see Feigelson et al. 2011), the predicted population in our Gum 31 field is 4085. 
In the lower panel of Fig. 12 we compare the Gum 31 XLF to the XLF for the CCCP sources as shown in Fig. 3 of Feigelson et al. (2011) (scaled to account for the difference in sample size). In the luminosity range $\log \left(L_{X}[\mathrm{erg} / \mathrm{s}]\right) \approx[31 \ldots 32]$, the distributions agree well. The lower number of very bright $\left(\log \left(L_{X}[\mathrm{erg} / \mathrm{s}]\right)>32\right)$ sources in the Gum 31 XLF seems to be related to the smaller number of $\mathrm{X}$-ray bright O-type stars.

\subsection{Clustered versus distributed population}

As mentioned above, the spatial distribution of the X-ray sources shows two prominent clusters, as well as a homogeneously distributed population. The sizes of the clustered and the distributed population of young stars can be estimated as follows. About 150 of the $\approx 500$ young stars are located in the clusters NGC 3324 or G286.38-0.26, or in one of the smaller clusterings discussed in Sect. 2.5. The remaining $\approx 350$ sources constitute the distributed population in the Gum 31 region. Their spatial distribution shows a rather uniform surface density and there is no obvious large-scale density gradient towards the edges of the observed area (above the expected level resulting from the decreasing instrumental sensitivity with increasing offaxis-angle). This suggests that this distributed population probably extends beyond the field of our Chandra observation. The clustered and the distributed population show also differences with respect to their stellar ages: while the clusters seem to be quite young ( $\$ 2 \mathrm{Myr}$ ), about $20 \%$ of the objects in the distributed population have ages of more than $\approx 3 \mathrm{Myr}$, up to about $10 \mathrm{Myr}$.

A similar bimodal spatial configuration was found for the $\mathrm{X}$-ray sources in the CCCP field (i.e., the more central parts of the Carina nebula): about half of the probable Carina members in the CCCP sample are members in one of several clusters, while the other half constitutes a so-called "widely-distributed population", which is spread out through the entire CCCP area (Feigelson et al. 2011). In Preibisch et al. (2011b) and Preibisch et al. (2011c) we found that this widely-distributed population of young stars shows a relatively wide range of stellar ages, up to $\sim 10 \mathrm{Myr}$.

These similarities raise the question, how the distributed population we find in the Gum 31 region is related to the widelydistributed population in the CCCP field. Using the numbers given in Feigelson et al. (2011), we find that the surface density ${ }^{13}$ of the widely-distributed population in the CCCP field is $\approx 1.15$ stars per square-arcminute. This predicts $\approx 330$ stars per single ACIS-I field, and this number is in good agreement with the size of the distributed population we find in our Gum 31 region.

It is thus feasible that the distributed population of young stars in Gum 31 is an extension of the widely-distributed population in the inner parts of the Carina nebula. Further implications of this assumption will be discussed in Sect. 9 .

\section{Diffuse X-ray emission}

The strong stellar winds of massive stars can fill the area around a star or a stellar cluster with a hot plasma that can be traced by diffuse soft X-ray emission. Such a diffuse X-ray component has been discovered in several high-mass star forming regions (see, e.g., Güdel et al. 2008; Townsley et al. 2011c). In the central

\footnotetext{
13 Table 1 of Feigelson et al. (2011) lists 5271 probable Carina members in the CCCP sample that are located outside the large-scale stellar enhancements A, B, C (which contain the different identified clusters). The area of this "Region D" is $\approx 1.27$ square-degrees.
}

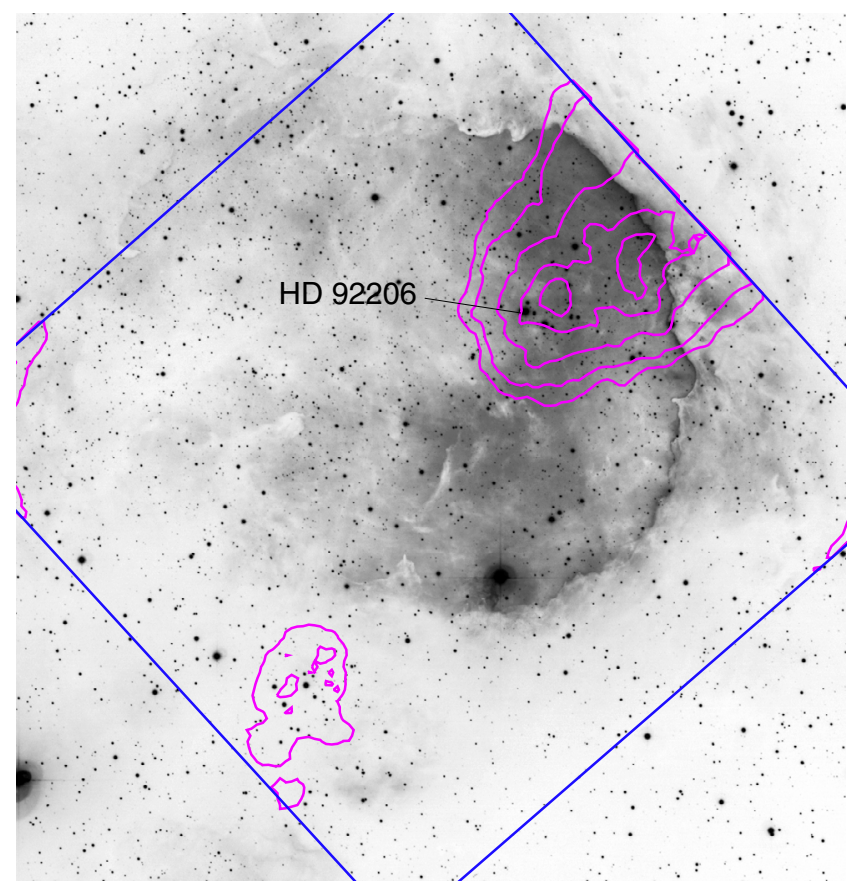

Fig. 13. Negative representation of the WFI $R$-band image (see www . eso.org/public/images/eso1207a/; image credit: ESO) with superposed contours of the smoothed apparent surface brightness of the diffuse X-ray emission in the $0.5-7 \mathrm{keV}$ band (point-sources have been excised). The contour levels are drawn at levels of $1.9,2.1,2.6,3.4$, and $4.0 \times 10^{-9}$ photons s $\mathrm{cm}^{-1} \mathrm{arcsec}^{-2}$. The blue lines mark the borders of the $17^{\prime} \times 17^{\prime}$ Chandra/ACIS-I field-of-view. The position of the O-star multiple system HD 92206 is marked.

parts of the CNC, strong diffuse X-ray emission has been detected in the CCCP survey and is described in detail in Townsley et al. $(2011 b, c)$.

We have performed a search for diffuse X-ray emission in the Gum 31 region in a similar way. We excised all point sources, then created X-ray images in several different energy bands and smoothed them with an adaptive-kernel smoothing code (Broos et al. 2010). The resulting smoothed diffuse X-ray image of Gum 31 for the $0.5-7 \mathrm{keV}$ band is shown in Fig. 13. One can clearly see significant levels of diffuse X-ray emission at several locations in Gum 31. The brightest patches of diffuse X-ray emission are found in a region just northwest of the stellar cluster NGC 3324. This suggests that the winds of the three O-type stars in NGC 3324 produce this hot plasma. The emission shows a very inhomogeneous spatial distribution, strongly concentrated to the northwest and much weaker in the other directions (as measured from the position of the cluster). The plasma flowing towards the northwest seems to be relatively well confined by the clouds surrounding the $\mathrm{H}$ II bubble, what leads to relatively high plasma densities and correspondingly strong X-ray emission at this location.

A second peak of diffuse X-ray emission is seen in the area of the partly embedded cluster G286.38-0.26. The origin of this plasma is not as easily explained, since no O-type stars are known to be present here.

A more detailed analysis of the diffuse X-ray emission in Gum 31 will be performed in connection with our new submm mapping data in a forthcoming publication. A detailed X-ray spectral analysis of the diffuse emission will allow us to estimate plasma temperatures and densities and provide more quantitative information. 


\section{Conclusions and summary}

Our Chandra observation of the Gum 31 region lead to the detection of $679 \mathrm{X}$-ray point sources, about 500 of which are young stars. This allowed us to identify, for the first time, a large sample of the young stellar population in the Gum 31 area, which is complete down to $\sim 1 M_{\odot}$. Extrapolation of the X-ray luminosity function down to $0.1 M_{\odot}$ suggests a total population of about 4000 young stars in the observed area. This shows that the still poorly explored northern parts of the CNC contain a very substantial fraction of the total young stellar population in the complex.

About $30 \%$ of the $\sim 500 \mathrm{X}$-ray detected young stars in the Gum 31 area are concentrated in the two very young $(\$ 2 \mathrm{Myr})$ clusters NGC 3324 and G286.38-0.26, but the large majority $(70 \%)$ is roughly uniformly distributed throughout the observed field-of-view. The analysis of the NIR color-magnitude diagram suggests that these distributed stars have a range of ages up to about 10 Myr.

Interestingly, the surface density of the distributed population in Gum 31 is similar to that of the "widely distributed population" that was found in the inner parts of the Carina nebula. This may suggest that the distributed population of young stars in the CNC extends over (at least) $100 \mathrm{pc}$. Such a large-scale distributed population would then contain several times more stars than the total number of members in all the known clusters (like $\operatorname{Tr} 14,15,16$, and as NGC 3324). These clusters clearly dominate the visual impression of the region, e.g., by creating the optically prominent $\mathrm{HII}$ regions. However, our results here suggest that most of the stellar mass is actually in the much more inconspicuous large-scale distributed stellar population. The CNC is thus a stellar association, in which the known clusters represent just a few compact subgroups. This is probably the consequence of the stochastic dynamical evolution (see, e.g., Parker et al. 2014) in combination with the extended history of star formation in the complex.

Further observations are required to quantify and finally evaluate this scenario. A spatially complete and deep enough $\mathrm{X}$-ray survey would be the ideal tool, but this is not really feasible. However, recent wide-field infrared surveys will soon provide more clues.

Acknowledgements. We thank the referee for a very careful and constructive report that helped to improve this paper. This work was supported by funding from Deutsche Forschungsgemeinschaft under DFG project number PR 569/9-1. Additional support came from funds from the Munich Cluster of Excellence "Origin and Structure of the Universe". The scientific results reported in this article are based on observations made by the Chandra X-ray Observatory. Support for this work was provided by the National Aeronautics and Space Administration through Chandra Award Number GO2-13010X issued by the Chandra X-ray Observatory Center, which is operated by the Smithsonian Astrophysical Observatory for and on behalf of the National Aeronautics Space Administration under contract NAS8-03060. This research has made use of software provided by the Chandra X-ray Center (CXC) in the application packages CIAO, ChIPS, and Sherpa. This work uses data from observations made with ESO Telescopes at the La Silla Paranal Observatory under program ID 088.C-0117(A). This work is based in part on observations made with the Spitzer Space Telescope, which is operated by the Jet Propulsion Laboratory, California Institute of Technology under a contract with NASA. This research has made use of the SIMBAD database, operated at CDS, Strasbourg, France.

\section{References}

Anders, E., \& Grevesse, N. 1989, Geochim. Cosmochim. Acta, 53, 197 Baraffe, I., Chabrier, G., Allard, F., \& Hauschildt, P. H. 1998, A\&A, 337, 403 Bessel, M. S., \& Brett, J. M. 1988, PASP, 100, 1134

Briceño, C., Preibisch, Th., Sherry, W., et al. 2007, in Protostars \& Planets V, eds

B. Reipurth, D. Jewitt, \& K. Keil (Tucson: University of Arizona Press), 345 Brooks, K. J., Cox, P., Schneider, N., et al. 2003, A\&A, 412, 751
Brooks, K. J., Garay, G., Nielbock, M., Smith, N., \& Cox, P. 2005, ApJ, 634, 436

Broos, P. S., Feigelson, E. D., Townsley, L. K., et al. 2007, ApJS, 169, 353

Broos, P. S., Townsley, L. K., Feigelson, E. D., et al. 2010, ApJ, 714, 1582

Broos, P. S., Townsley, L. K., Feigelson, E. D., et al. 2011a, ApJS, 194, 2

Broos, P. S., Getman, K. V., Povich, M. S., et al. 2011b, ApJS, 194, 4

Broos, P., Townsley, L., Getman, K., \& Bauer, F. 2012, Astrophysics Source Code Library, 3001

Campillay, A., Arias, J., Barba, R., et al. 2007, in VI Reunion Anual Sociedad Chilena de Astronomia (SOCHIAS), 63

Cappa, C., Niemela, V. S., Amorín, R., \& Vasquez, J. 2008, A\&A, 477, 173

Carraro, G., Patat, F., \& Baumgardt, H. 2001, A\&A, 371, 107

Casertano, S., \& Hut, P. 1985, ApJ, 298, 80

Dalton, G. B., Caldwell, M., Ward, A. K., et al. 2006, Proc. SPIE, 6269, 30

Dias, W. S., Alessi, B. S., Moitinho, A., \& Lépine, J. R. D. 2002, A\&A, 389, 871

Dutra, C. M., Bica, E., Soares, J., \& Barbuy, B. 2003, A\&A, 400, 533

Emerson, J., McPherson, A., \& Sutherland, W. 2006, The Messenger, 126, 41

Fedele, D., van den Ancker, M. E., Henning, T., Jayawardhana, R., \& Oliveira, J. M. 2010, A\&A, 510, A72

Feigelson, E. D., Townsley, L., Güdel, M., \& Stassun, K. 2007, in Protostars \& Planets V, eds. B. Reipurth, D. Jewitt, \& K. Keil (Univ. Arizona Press), 313 Feigelson, E. D., Getman, K. V., Townsley, L. K., et al. 2011, ApJS, 194, 9

Freeman, P., Doe, S., \& Siemiginowska, A. 2001, Proc. SPIE, 4477, 76

Freeman, P. E., Kashyap, V., Rosner, R., \& Lamb, D. Q. 2002, ApJS, 138, 185

Gaczkowski, B., Preibisch, T., Ratzka, T., et al. 2013, A\&A, 549, A67

Gagné, M., Fehon, G., Savoy, M. R., et al. 2011, ApJS, 194, 5

Garmire, G. P., Bautz, M. W., Ford, P. G., Nousek, J. A., \& Ricker, G. R., Jr. 2003, Proc. SPIE, 4851, 28

Gehrels, N. 1986, ApJ, 303, 336

Getman, K. V., Feigelson, E. D., Grosso, N., et al. 2005, ApJS, 160, 353

Getman, K. V., Feigelson, E. D., Broos, P. S., Townsley, L. K., \& Garmire, G. P. 2010, ApJ, 708, 1760

Getman, K. V., Broos, P. S., Feigelson, E. D., et al. 2011, ApJS, 194, 3

Güdel, M., Briggs, K. R., Arzner, K., et al. 2007, A\&A, 468, 353

Güdel, M., Briggs, K. R., Montmerle, T., et al. 2008, Science, 319, 309

Gum, C. S. 1955, Mem. Roy. Astron. Soc., 67, 155

Gutermuth, R. A., Megeath, S. T., Myers, P. C., et al. 2009, ApJS, 184, 18

Irwin, M. J., Lewis, J., Hodgkin, S., et al. 2004, Proc. SPIE, 5493, 411

Kroupa, P. 2002, Science, 295, 82

Lejeune T., \& Schaerer, D. 2001, A\&A, 366, 538

Lucy, L. B. 1974, AJ, 79, 745

Martins, F., Schaerer, D., \& Hillier, D. J. 2005, A\&A, 436, 1049

Mathys, G. 1988, A\&AS, 76, 427

O’Connell, D. J. K. 1956, Ricerche Astronomiche, 3, 313

Ohlendorf, H., Preibisch, T., Gaczkowski, B., et al. 2012, A\&A, 540, A81

Ohlendorf, H., Preibisch, T., Gaczkowski, B., et al. 2013, A\&A, 552, A14

Ohlendorf, H., Preibisch, T., Roccatagliata, V., \& Ratzka, T. 2014, AJ, submitted

Parker, R. J., Wright, N. J., Goodwin, S. P., \& Meyer, M. R. 2014, MNRAS, 438, 620

Povich, M. S., Smith, N., Majewski, S. R., et al. 2011, ApJS, 194, 14

Preibisch, T. 2012, Res. Astron. Astrophys., 12, 1

Preibisch, T., \& Feigelson, E. D. 2005, ApJS, 160, 390

Preibisch, Th., Kim, Y.-C., Favata, F., et al. 2005, ApJS, 160, 401

Preibisch, T., Schuller, F., Ohlendorf, H., et al. 2011a, A\&A, 525, A92

Preibisch, T., Hodgkin, S., Irwin, M., et al. 2011b, ApJS, 194, 10

Preibisch, T., Ratzka, T., Kuderna, B., et al. 2011c, A\&A, 530, A34

Preibisch, T., Roccatagliata, V., Gaczkowski, B., \& Ratzka, T. 2012, A\&A, 541, A132

Roccatagliata, V., Preibisch, T., Ratzka, T., \& Gaczkowski, B. 2013, A\&A, 554, A6 Siess, L., Dufour, E., \& Forestini, M. 2000, A\&A, 358, 593

Smith, N. 2006, MNRAS, 367, 763

Smith, N., \& Brooks, K. J. 2007, MNRAS, 379, 1279

Smith, N., \& Brooks, K. J. 2008, in Handbook of Star Forming Regions, Vol. II:

The Southern Sky, ASP Monograph Publications, 5 (ed. B. Reipurth), 138

Smith, N., Bally, J., \& Walborn, N. R. 2010a, MNRAS, 405, 1153

Smith, N., Povich, M. S., Whitney, B. A., et al. 2010b, MNRAS, 406, 952

Stassun, K. G., van den Berg, M., Feigelson, E., \& Flaccomio, E. 2006, ApJ, 649, 914

Stelzer, B., Flaccomio, E., Montmerle, T., et al. 2005, ApJS, 160, 557

Townsley, L., Broos, P., Bauer, F., \& Getman, K. 2003, BAAS, 35, 644 Townsley, L. K., Broos, P. S., Corcoran, M. F., et al. 2011a, ApJS, 194, 1 Townsley, L. K., Broos, P. S., Chu, Y.-H., et al. 2011b, ApJS, 194, 15 Townsley, L. K., Broos, P. S., Chu, Y.-H., et al. 2011c, ApJS, 194, 16 Walborn, N. R. 1982, AJ, 87, 1300

Weisskopf, M. C., Brinkman, B., Canizares, C., et al. 2002, PASP, 114, 1 Wang, J., Feigelson, E. D., Townsley, L. K., et al. 2011, ApJS, 194, 11 Wolk, S. J., Harnden, F. R., Jr., Flaccomio, E., et al. 2005, ApJS, 160, 423 Wolk, S. J., Broos, P. S., Getman, K. V., et al. 2011, ApJS, 194, 12 